OPTIMAL CONTROL OF A SEMILINEAR PDE WITH NONLOCAL RADIATION INTERFACE CONDITIONS

\author{
By
}

C. Meyer

P. Philip

and

F. Tröltzsch

IMA Preprint Series \# 2002

(October 2004)

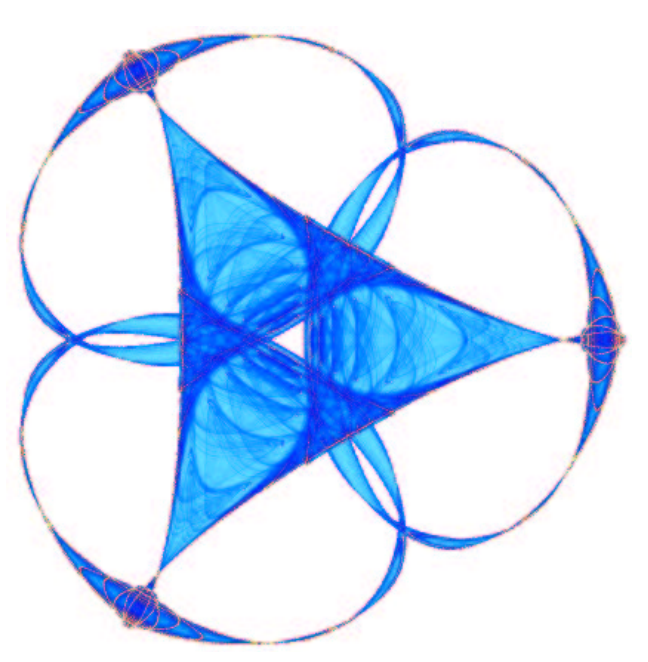

INSTITUTE FOR MATHEMATICS AND ITS APPLICATIONS UNIVERSITY OF MINNESOTA

514 Vincent Hall 206 Church Street S.E. Minneapolis, Minnesota 55455-0436 Phone: 612/624-6066 Fax: 612/626-7370 URL: http://www.ima.umn.edu 


\title{
OPTIMAL CONTROL OF A SEMILINEAR PDE WITH NONLOCAL RADIATION INTERFACE CONDITIONS*
}

\author{
C. MEYER ${ }^{\dagger}$, P. PHILIP ${ }^{\ddagger}$, AND F. TRÖLTZSCH ${ }^{\dagger}$
}

\begin{abstract}
We consider a control constrained optimal control problem governed by a semilinear elliptic equation with nonlocal interface conditions. These conditions occur during the modeling of diffuse-gray conductive-radiative heat transfer. The problem arises from the aim to optimize the temperature gradient within crystal growth by the physical vapor transport (PVT) method. Based on a minimum principle for the semilinear equation as well as $L^{\infty}$-estimates for the weak solution, we establish the existence of an optimal solution as well as necessary optimality conditions. The theoretical results are illustrated by results of numerical computations.
\end{abstract}

Key words. Optimal control, semilinear elliptic equations, nonlocal interface conditions, boundedness of solutions

AMS subject classifications. 49K20, 35J65, 49J20, 80M50

1. Introduction. In this paper, we investigate an optimal control problem related to the sublimation growth of silicon carbide single crystals $(\mathrm{SiC})$ by the physical vapor transport (PVT) method. The semiconductor material $\mathrm{SiC}$ is used in numerous industrial applications, e.g. the production of optoelectronic devices such as blue and green LEDs and lasers. For the PVT method, polycrystalline SiC powder is placed under a low-pressure inert gas atmosphere at the bottom of a cavity inside a graphite crucible. The crucible is heated up to temperatures between 2000 and $3000 \mathrm{~K}$ by induction. Due to the high temperatures and the low pressure, the SiC powder sublimates and crystallizes at a single-crystalline $\mathrm{SiC}$ seed located at the cooled top of the cavity, such that the single crystal grows into the reaction chamber. See $[7,8]$ for more details.

Here, we neglect the electromagnetic induction problem and focus on the conductiveradiative heat transfer in the growth apparatus. Therefore, we consider a simplified setup of the growth apparatus, shown in Fig. 1.1, where $\Omega_{\mathrm{s}}$ denotes the domain of the solid graphite crucible, whereas $\Omega_{\mathrm{g}}$ is the domain of gas phase inside.

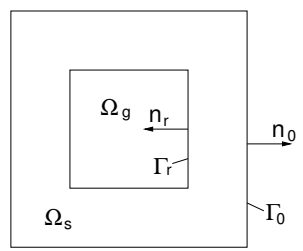

FIG. 1.1. 2-dimensional section through an exemplary domain for nonlocal radiative heat transfer.

\footnotetext{
*Supported by the DFG Research Center "Mathematics for key technologies" (FZT 86) in Berlin.

†Institut für Mathematik, Technische Universität Berlin, D-10623 Berlin, Str. des 17. Juni 136, Germany.

${ }^{\ddagger}$ Institute for Mathematics and its Applications (IMA), University of Minnesota, 400 Lind Hall, 207 Church Street S.E., Minneapolis, MN 55455-0436, USA.

$\S$ Supported by the Weierstrass Institute for Applied Analysis and Stochastics (WIAS) in Berlin.
} 
A very important determining factor for the crystal's quality and growth rate are the temperature distribution in the gas phase and, especially, the temperature gradient close to the surface of the growing crystal [12]. Since we do not consider the electromagnetic induction, we will optimize the temperature gradient in the gas phase $\Omega_{\mathrm{g}}$ by directly controlling the heat source $u$ in $\Omega_{\mathrm{s}}$.

The temperature $y$ inside the growth apparatus arises as the solution of the conductive-radiative heat transfer problem in the growth apparatus. Accounting for radiative contributions is essential owing to the high temperatures. Thus, the problem is described by the stationary heat equation with radiation interface and boundary conditions on $\Gamma_{\mathrm{r}}$ and $\Gamma_{0}$, respectively. We take $\Omega_{\mathrm{s}}$ to be entirely opaque, whereas $\Omega_{\mathrm{g}}$ represents a transparent medium which does not interact with radiation. Furthermore, the radiative surfaces $\Gamma_{0}:=\partial \Omega$ and $\Gamma_{\mathrm{r}}:=\bar{\Omega}_{\mathrm{s}} \cap \bar{\Omega}_{\mathrm{g}}$ are presumed to be diffuse-gray, i.e. the emissivity $\varepsilon$ is independent of both the direction and the wavelength of the radiation. In particular, the local radiative heat exchange on $\Gamma_{0}$ can be modeled by the Boltzmann radiation condition with an external temperature $y_{0}$. Due to the heat exchange between points on $\Gamma_{\mathrm{r}}$, we obtain an additional radiative heat flux on $\Gamma_{\mathrm{r}}$, denoted by $q_{\mathrm{r}}$.

In addition to the stationary semilinear heat equation with radiation interface and boundary conditions, we consider box constraints for the control function $u$. Thus, the optimal control problem, considered here, reads as follows:

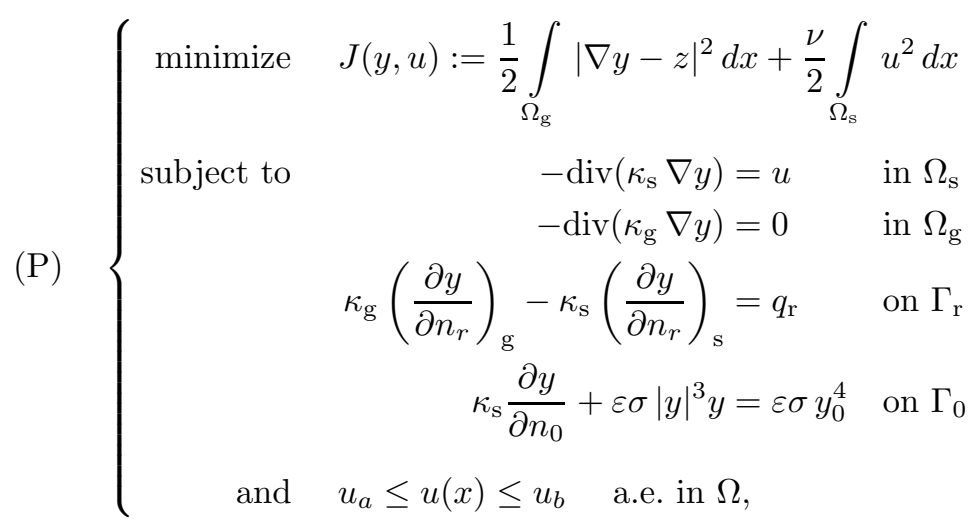

where $n_{0}$ is the outward unit normal on $\Gamma_{0}$, and $n_{\mathrm{r}}$ is the unit normal on $\Gamma_{\mathrm{r}}$ facing outward with respect to $\Omega_{\mathrm{s}}$ (cf. Fig. 1.1). Furthermore, $z$ denotes the desired temperature gradient and $\nu>0$ is a Tikhonov regularization parameter. In the state equation, $\sigma$ represents the Boltzmann radiation constant, and $\kappa_{\mathrm{s}}, \kappa_{\mathrm{g}}$ denote the thermal conductivities in $\Omega_{\mathrm{s}}, \Omega_{\mathrm{g}}$, respectively.

In contrast to the boundary condition on $\Gamma_{0}$, the radiative heat transfer on $\Gamma_{\mathrm{r}}$ is nonlocal. The corresponding mathematical model used here is described in detail, e.g., in $[11,14]$. It provides the additional radiative heat flux $q_{\mathrm{r}}$ on $\Gamma_{\mathrm{r}}$ given by

$$
q_{\mathrm{r}}=(I-K)(I-(1-\varepsilon) K)^{-1} \varepsilon \sigma|y|^{3} y:=G \sigma|y|^{3} y,
$$

where $K$ is an integral operator representing the irradiation on $\Gamma_{\mathrm{r}}$. The nonlocal operators $K$ and $G$ will be specified in Section 3.1. The nonlocal radiation on $\Gamma_{\mathrm{r}}$ represents the main characteristic of our problem, since the nonlinearity in the state equation in $(\mathrm{P})$ is in general not monotone due to nonpositivity of $G$ (see Section 3 and $[14])$. 
The state equation in $(\mathrm{P})$ is of semilinear elliptic type. Therefore, the optimal control problem can be viewed as a semilinear elliptic optimal control problem. The list of publications in this field is already quite extensive. We only mention Casas [3] or Bonnans and Casas [2], who consider the Pontryagin principle, or Casas, Tröltzsch and Unger [5], Bonnans [1], Casas and Mateos [4], who consider different aspects of second-order sufficient optimality conditions. This list might be extended considerably by including associated papers on different aspects of numerical analysis.

Our paper differs from all these contributions by the interface conditions containing the nonmonotone and nonlocal operator $G$. Therefore, our boundary value problem is of nonmonotone type so that special techniques must be applied.

The paper is organized as follows: After stating the mathematical setting in Section 2 , we provide auxiliary results on the nonlocal operator $G$ as well as a general boundedness result for a class of nonlinear equations, see Section 3. In Section 4, we prove a weak maximum principle for the semilinear state equation and an $L^{\infty}$-estimate for its solutions, followed by the existence of an optimal solution in Section 5. Section 6 is devoted to the existence and boundedness of a solution to the linearized equation. In Section 7, we establish first order necessary optimality conditions based on the differentiability of the solution operator associated with the semilinear equation. The corresponding adjoint state is introduced at the end of Section 7. Finally, Section 8 presents some numerical results.

2. The mathematical setting. Throughout this paper, we assume the following conditions (A1) - (A3) on the domain $\Omega$ and on the quantities and functions occurring in $(\mathrm{P})$ :

(A1) We assume that $\Omega \subset \mathbb{R}^{3}$ is a bounded simply connected domain with Lipschitz boundary $\Gamma_{0}$. The boundary of the simply connected subdomain $\bar{\Omega}_{\mathrm{g}} \subset \Omega$, denoted by $\Gamma_{\mathrm{r}}$, is assumed to be a closed Lipschitz surface that is piecewise $C^{1, \delta}$. Notice that the distance of $\Gamma_{\mathrm{r}}$ to $\Gamma_{0}$ is positive. Then, $\Omega_{\mathrm{s}}$ is defined by $\Omega_{\mathrm{s}}=\Omega \backslash \bar{\Omega}_{\mathrm{g}}$ (cf. Fig. 1.1).

(A2) The Boltzmann radiation constant is assumed to be positive, i.e. $\sigma \in \mathbb{R}^{+}$. For the thermal conductivity, we assume $\kappa \in L^{\infty}(\Omega)$ with

$$
\kappa(x)=\left\{\begin{array}{l}
\kappa_{\mathrm{s}}(x) \text { in } \Omega_{\mathrm{s}} \\
\kappa_{\mathrm{g}}(x) \text { in } \Omega_{\mathrm{g}}
\end{array}\right.
$$

and $\kappa(x) \geq \kappa_{\min }>0$ a.e. on $\Omega$. Furthermore, the emissivity $\varepsilon \in L^{\infty}\left(\Gamma_{0} \cup \Gamma_{\mathrm{r}}\right)$ is bounded by $1 \geq \varepsilon \geq \varepsilon_{\min }>0$ a.e. on $\Gamma_{0} \cup \Gamma_{\mathrm{r}}$.

(A3) The desired temperature gradient $z$ is given in $L^{2}\left(\Omega_{\mathrm{g}}\right)$ and $\nu$ is a positive constant. For the box constraints, we assume $u_{a}, u_{b} \in L^{\infty}\left(\Omega_{\mathrm{s}}\right)$ and $0 \leq u_{a}(x)<$ $u_{b}(x)$ a.e. in $\Omega_{\mathrm{s}}$.

Notation. For a given $p$ with $1 \leq p \leq \infty$, an operator $B: L^{p}\left(\Gamma_{\mathrm{r}}\right) \rightarrow L^{p}\left(\Gamma_{\mathrm{r}}\right)$ is said to be positive, if $v \in L^{p}\left(\Gamma_{\mathrm{r}}\right)$ and $v \geq 0$ a.e. on $\Gamma_{\mathrm{r}}$ imply $B v \geq 0$ a.e. on $\Gamma_{\mathrm{r}}$. Furthermore, 1 denotes the function $e(x) \equiv 1$ a.e., and $I$ is the identity operator in the respective function spaces. We introduce the set of admissible controls by $U_{\mathrm{ad}}:=\left\{u \in L^{\infty}\left(\Omega_{\mathrm{s}}\right) \mid u_{a}(x) \leq u(x) \leq u_{b}(x)\right.$ a.e. in $\left.\Omega_{\mathrm{s}}\right\}$. The measure of a certain set $A \subset \mathbb{R}^{n}, n \geq 1$ is denoted by $|A|$, and $\tau_{\mathrm{r}}$ denotes the trace operator on $\Gamma_{\mathrm{r}}$, whereas $\tau_{0}$ is the trace on $\Gamma_{0}$. Throughout this paper, $c$ is a generic constant.

Let $W$ be a Banach space with its dual space $W^{*}$. Then, for $f \in W$ and $g \in W^{*}$, $\langle f, g\rangle$ denotes the associated pairing. 
3. Auxiliary results. In Section 3.1, we present some properties of the nonlocal radiation operator $G$. Since $G$ is in general not positive, the nonlinearity in the semilinear state equation is nonmonotone. However, $G$ still has sufficiently benign properties such that the nonlinear parts in the state equation are pseudomonotone (see [10]). These properties of $G$ are also used to prove the boundedness of the solution in Section 4 below.

In Lemma 3.7 of Section 3.2, we will prove the boundedness of the solution $y$ for a general class of nonlinear equations that applies to both the semilinear case and its linearized version.

3.1. The nonlocal radiation operator. The operators $K$ and $G$ arising from the nonlocal radiation on $\Gamma_{\mathrm{r}}$ were investigated in detail by Laitinen and Tiihonen $[10,14,15]$. We recall some of their results for convenience:

DeFinition 3.1. The integral operator $K$, representing the irradiation on $\Gamma_{\mathrm{r}}$, is given by

$$
(K y)(x)=\int_{\Gamma_{\mathrm{r}}} \omega(x, z) y(z) d s_{z}
$$

with a symmetric kernel $\omega$ which is, in the two-dimensional case, defined by

$$
\omega(x, z)=\Xi(x, z) \frac{\left[n_{\mathrm{r}}(z) \cdot(x-z)\right]\left[n_{\mathrm{r}}(x) \cdot(z-x)\right]}{2|z-x|^{3}},
$$

and, in the three-dimensional case, defined by

$$
\omega(x, z)=\Xi(x, z) \frac{\left[n_{\mathrm{r}}(z) \cdot(x-z)\right]\left[n_{\mathrm{r}}(x) \cdot(z-x)\right]}{\pi|z-x|^{4}},
$$

where $x, z$ denote two points on $\Gamma_{\mathrm{r}}$, and $n_{\mathrm{r}}(x)$ is the unit normal at $x$ facing outward with respect to $\Omega_{\mathrm{s}}$ (see Fig. 1.1). Here, $\Xi$ represents the visibility factor which is given by

$$
\Xi(x, z)= \begin{cases}0 & \text { if } \overline{x z} \cap \Omega_{\mathrm{g}} \neq \emptyset \\ 1 & \text { if } \overline{x z} \cap \Omega_{\mathrm{g}}=\emptyset .\end{cases}
$$

In [15], it is proven that $\omega(x, z)$ can have a singularity at $x$ of type $|x-z|^{-(1-\delta)}$ in the two-dimensional and $|x-z|^{-2(1-\delta)}$ in the three-dimensional case, which is, in both cases, integrable. This is the key point to the following lemma also derived in [15].

LEMMA 3.2 .

(i) $K$ maps $L^{p}\left(\Gamma_{\mathrm{r}}\right)$ to $L^{p}\left(\Gamma_{\mathrm{r}}\right)$ for all $1 \leq p \leq \infty$.

(ii) If $\Gamma_{\mathrm{r}}$ is a closed Lipschitz surface that is piecewise $C^{1, \delta}$, then $K \mathbf{1}=\mathbf{1}$ holds a.e. on $\Gamma_{\mathrm{r}}$.

(iii) The operator $I-(1-\varepsilon) K: L^{p}\left(\Gamma_{\mathrm{r}}\right) \rightarrow L^{p}\left(\Gamma_{\mathrm{r}}\right)$ is invertible.

With the help of Lemma 3.2, Tiihonen and Laitinen proved the following properties of $G=(I-K)(I-(1-\varepsilon) K)^{-1} \varepsilon$ (cf. [14, Lemma 6] and [10, Lemma 8]).

LEMMA 3.3.

(i) $G$ is a bounded linear operator from $L^{p}\left(\Gamma_{\mathrm{r}}\right)$ to itself for all $1 \leq p \leq \infty$.

(ii) $G$ can be written as $G=I-H$ with a positive operator $H$.

(iii) For all $y \in L^{5}\left(\Gamma_{\mathrm{r}}\right), \int_{\Gamma_{\mathrm{r}}} G\left(\sigma|y|^{3} y\right) y d s \geq 0$ holds true. 
Here, we show another property of $G$ that we will use subsequently:

LEMMA 3.4. For every function $v(x) \equiv k=$ const. a.e. on $\Gamma_{\mathrm{r}}$, we have $G^{*} v=0$.

Proof: Since $K$ has a symmetric kernel, $K$ is formally self-adjoint, i.e. $\langle v, K w\rangle=$ $\langle K v, w\rangle$ for all $v \in L^{p}\left(\Gamma_{\mathrm{r}}\right)$ and $w \in L^{q}\left(\Gamma_{\mathrm{r}}\right), 1 / p+1 / q=1$. Thus, together with Lemma 3.2, (ii), we obtain $K^{*} \mathbf{1}=\mathbf{1}$ a.e. on $\Gamma_{\mathrm{r}}$. Therefore, with the definition of $G$, we find

$$
G^{*} v=\varepsilon\left(I-K^{*}(1-\varepsilon)\right)^{-1}\left(I-K^{*}\right) v=\varepsilon\left(I-K^{*}(1-\varepsilon)\right)^{-1}(k-k)=0 .
$$

3.2. Boundedness for a nonlinear equation. We have to show the boundedness of the solution of the semilinear state equation and its linearization. To unify the proofs, we first prove an auxiliary result for a nonlinear equation of the form

$$
\langle F(y), v\rangle=\langle f, v\rangle \quad \forall v \in W
$$

where $F: W \rightarrow W^{*}$ is a certain mapping, and $y$ is a given solution in a space $W \subseteq H^{1}(\Omega)$. It will be shown that, under suitable assumptions, a solution $y$ belongs to the function space

$$
V^{\infty}:=H^{1}(\Omega) \cap L^{\infty}(\Omega)
$$

equipped with the norm

$$
\|v\|_{V^{\infty}}=\|v\|_{H^{1}(\Omega)}+\|v\|_{L^{\infty}(\Omega)} .
$$

REMARK 3.5. If $y \in V^{\infty}$, then $\tau_{\mathrm{r}} y \in L^{\infty}\left(\Gamma_{\mathrm{r}}\right)$, and $\tau_{0} y \in L^{\infty}\left(\Gamma_{0}\right)$.

This is an immediate consequence of the following Lemma 3.6 that follows from Proposition 5.2, part (ii) in [6].

LEMMA 3.6. For a bounded Lipschitz domain $\Omega \subset \mathbb{R}^{n}, n \in \mathbb{N}$, the trace operator $\tau: H^{1}(\Omega) \rightarrow H^{1 / 2}(\partial \Omega)$ is positive, i.e. $\tau v \geq 0$ a.e. on $\partial \Omega$ for $v \geq 0$ a.e. on $\Omega$.

To show the boundedness of a solution to (3.3), we use the following hypotheses:

HYPOTHESES FOR THE NONLINEAR EQUATION (3.3):

(H1) (Regularity of the inhomogeneity): The right-hand side of (3.3) can be expressed as

$$
\langle f, v\rangle=\int_{\Omega} f_{\Omega} v d x+\int_{\Gamma_{\mathrm{r}}} f_{\mathrm{r}} v d s+\int_{\Gamma_{0}} f_{0} v d s,
$$

where $f_{\Omega}, f_{\mathrm{r}}$, and $f_{0}$ satisfy $f_{\Omega} \in L^{p_{1}}(\Omega), f_{\mathrm{r}} \in L^{p_{2}}\left(\Gamma_{\mathrm{r}}\right)$, and $f_{0} \in L^{p_{2}}\left(\Gamma_{0}\right)$, with $p_{1}=6 /(5-s), p_{2}=4 /(3-s)$ and $1<s<3$.

(H2) (Coercivity): There is a constant $k_{0} \geq 0$ such that, for the given solution $y \in W$ and for each $k>k_{0}$, the functions

$$
\varphi_{k}(x):=\left\{\begin{array}{c}
y(x)-k, \quad y(x) \geq k \\
0, \quad|y(x)|<k \\
y(x)+k, \quad y(x) \leq-k,
\end{array}\right.
$$

are elements of $W$, and there is a constant $c>0$ such that the nonlinearity in (3.3) satisfies, for each $k>k_{0}$ :

$$
\left\langle F(y), \varphi_{k}\right\rangle \geq c\left\|\varphi_{k}\right\|_{H^{1}(\Omega)}^{2} .
$$


Lemma 3.7. Suppose that $\Omega \subset \mathbb{R}^{3}$ satisfies (A1), that $y \in W$ is a solution to (3.3) and that the Hypotheses $(\mathrm{H} 1)$ and $(\mathrm{H} 2)$ are fulfilled. Then $y \in V^{\infty}$, and there exists a constant $c_{0}$ only depending on $\Omega$ such that

$$
\|y\|_{L^{\infty}(\Omega)}+\|y\|_{L^{\infty}\left(\Gamma_{\mathrm{r}} \cup \Gamma_{0}\right)} \leq c_{0}\left(k_{0}+\left\|f_{\Omega}\right\|_{L^{p_{1}}(\Omega)}+\left\|f_{\mathrm{r}}\right\|_{L^{p_{2}\left(\Gamma_{\mathrm{r}}\right)}}+\left\|f_{0}\right\|_{L^{p_{2}\left(\Gamma_{0}\right)}}\right)
$$

holds true.

Proof: The proof is based on a technique introduced by Stampacchia and Kinderlehrer $[6,13]$ in the linear case. For given $k>k_{0}$, we define $A_{\Omega}(k):=\{x \in \Omega|| y(x) \mid \geq k\}$ and introduce $A_{\mathrm{r}}(k)$ and $A_{0}(k)$ analogously. Our aim is to show that there is a $k>0$ with $\left|A_{\Omega}(k)\right|=\left|A_{\mathrm{r}}(k)\right|=\left|A_{0}(k)\right|=0$. We start with Hypothesis (H2), that yields

$$
\begin{aligned}
& \left\langle F(y), \varphi_{k}\right\rangle \geq c\left\|\varphi_{k}\right\|_{H^{1}(\Omega)}^{2} \geq c\left(\left\|\varphi_{k}\right\|_{L^{6}(\Omega)}^{2}+\left\|\varphi_{k}\right\|_{L^{4}\left(\Gamma_{\mathrm{r}}\right)}^{2}+\left\|\varphi_{k}\right\|_{L^{4}\left(\Gamma_{0}\right)}^{2}\right) \\
& \geq c\left(\left(\int_{A_{\Omega}(k)}(|y|-k)^{6} d x\right)^{1 / 3}+\left(\int_{A_{\mathrm{r}}(k)}(|y|-k)^{4} d s\right)^{1 / 2}+\left(\int_{A_{0}(k)}(|y|-k)^{4} d s\right)^{1 / 2}\right),
\end{aligned}
$$

since $\varphi_{k}$ equals 0 on $\Omega \backslash A_{\Omega}(k)$, on $\Gamma_{\mathrm{r}} \backslash A_{\mathrm{r}}(k)$, and on $\Gamma_{0} \backslash A_{0}(k)$, respectively. If $h>k>k_{0} \geq 0$, then $A(h) \subseteq A(k)$, where $A$ stands generally for $A_{\Omega}, A_{\mathrm{r}}$, and $A_{0}$, and we have for an arbitrary $m \in \mathbb{N}$ :

$$
\left(\int_{A(k)}(|y|-k)^{m} d x\right)^{2 / m} \geq\left(\int_{A(h)}(h-k)^{m} d x\right)^{2 / m}=(h-k)^{2}|A(h)|^{2 / m} .
$$

Defining

$$
\psi(h):=\left|A_{\Omega}(h)\right|^{1 / 3}+\left|A_{0}(h)\right|^{1 / 2}+\left|A_{\mathrm{r}}(h)\right|^{1 / 2},
$$

one obtains

$$
\left\|\varphi_{k}\right\|_{H^{1}(\Omega)}^{2} \geq c(h-k)^{2} \psi(h) .
$$

Now, we investigate the right-hand side in (3.3). Hypothesis (H1), Hölder's inequality, and embedding theorems imply

$$
\begin{aligned}
\left\langle f, \varphi_{k}\right\rangle \leq & \left\|f_{\Omega}\right\|_{L^{6 / 5}\left(A_{\Omega}(k)\right)}\left\|\varphi_{k}\right\|_{L^{6}(\Omega)}+\left\|f_{\mathrm{r}}\right\|_{L^{4 / 3}\left(A_{\mathrm{r}}(k)\right)}\left\|\varphi_{k}\right\|_{L^{4}\left(\Gamma_{\mathrm{r}}\right)} \\
& \quad+\left\|f_{0}\right\|_{L^{4 / 3}\left(A_{0}(k)\right)}\left\|\varphi_{k}\right\|_{L^{4}\left(\Gamma_{0}\right)} \\
\leq & c\left(\left\|f_{\Omega}\right\|_{L^{6 / 5}\left(A_{\Omega}(k)\right)}+\left\|f_{\mathrm{r}}\right\|_{L^{4 / 3}\left(A_{\mathrm{r}}(k)\right)}+\left\|f_{0}\right\|_{L^{4 / 3}\left(A_{0}(k)\right)}\right)\left\|\varphi_{k}\right\|_{H^{1}(\Omega)} .
\end{aligned}
$$

Applying (H2), (3.3), and Young's inequality, we obtain

$$
\left\|\varphi_{k}\right\|_{H^{1}(\Omega)}^{2} \leq c\left(\left\|f_{\Omega}\right\|_{L^{6 / 5}\left(A_{\Omega}(k)\right)}^{2}+\left\|f_{\mathrm{r}}\right\|_{L^{4 / 3}\left(A_{\mathrm{r}}(k)\right)}^{2}+\left\|f_{0}\right\|_{L^{4 / 3}\left(A_{0}(k)\right)}^{2}\right) .
$$

Using again Hölder's inequality, the first norm on the right-hand side can be estimated by

$$
\begin{aligned}
\left(\int_{A_{\Omega}(k)} f_{\Omega}^{6 / 5} d x\right)^{5 / 3} & \leq\left(\left\|f_{\Omega}^{6 / 5}\right\|_{L^{5 /(5-s)}\left(A_{\Omega}(k)\right)}\left|A_{\Omega}(k)\right|^{s / 5}\right)^{5 / 3} \\
& \leq\left\|f_{\Omega}\right\|_{L^{p_{1}}(\Omega)}^{2}\left|A_{\Omega}(k)\right|^{s / 3}
\end{aligned}
$$


with $p_{1}=6 /(5-s)$ and $1<s<3$ as defined above. Similarly, we derive

$$
\left(\int_{A_{\mathrm{r}}(k)} f_{\mathrm{r}}^{4 / 3} d x\right)^{3 / 2} \leq\left\|f_{\mathrm{r}}\right\|_{L^{p_{2}\left(\Gamma_{\mathrm{r}}\right)}}^{2}\left|A_{\mathrm{r}}(k)\right|^{s / 2}
$$

with $p_{2}=4 /(3-s)$ (and analogously on $\left.A_{0}\right)$.

Due to the Taylor expansion, we have for three abritrary real nonnegative numbers $a, b$, and $c$ that $\left(a^{s}+b^{s}+c^{s}\right) \leq(a+b+c)^{s}$ for all $s>1$. Choosing $a=\left|A_{\Omega}(k)\right|^{1 / 3}$, $b=\left|A_{\mathrm{r}}(k)\right|^{1 / 2}$, and $c=\left|A_{0}(k)\right|^{1 / 2}$, combining (3.10), (3.11), and (3.9) yields

$$
\left\|\varphi_{k}\right\|_{H^{1}(\Omega)}^{2} \leq c \varrho_{f} \psi(k)^{s}
$$

with $\varrho_{f}=\left\|f_{\Omega}\right\|_{L^{p_{1}(\Omega)}}^{2}+\left\|f_{\mathrm{r}}\right\|_{L^{p_{2}\left(\Gamma_{\mathrm{r}}\right)}}^{2}+\left\|f_{0}\right\|_{L^{p_{2}\left(\Gamma_{0}\right)}}^{2}$, and $\psi$ as defined in (3.7). Together with (3.8), it follows that

$$
\psi(h) \leq \frac{c \varrho_{f}}{(h-k)^{2}} \psi(k)^{s}, \quad \text { for } h>k>k_{0} \geq 0 .
$$

Stampacchia proved in [13] that each nonnegative and nonincreasing function $\psi=\psi(t)$ satisfying (3.12) with some $s>1$, has a zero at some $t=d, d>0$ where

$$
d=k_{0}+2^{s /(s-1)}\left(c\left|\psi\left(k_{0}\right)\right|^{s-1}\right)^{1 / 2} \sqrt{\varrho_{f}},
$$

and, in our case, $\left|\psi\left(k_{0}\right)\right|=\psi\left(k_{0}\right)$ can be estimated by

$$
\psi\left(k_{0}\right) \leq \psi(0)=|\Omega|^{1 / 3}+\left|\Gamma_{0}\right|^{1 / 2}+\left|\Gamma_{\mathrm{r}}\right|^{1 / 2}
$$

(see also Kinderlehrer and Stampacchia [6, Lemma B.1]). Due to the definition of $\psi$ in (3.7), this implies $|y(x)| \leq d$ a.e. on $\Omega$ and on $\Gamma_{\mathrm{r}} \cup \Gamma_{0}$. Thus, with the definition of $\varrho_{f}$, we obtain

$$
\begin{aligned}
\|y\|_{L^{\infty}(\Omega)}+\|y\|_{L^{\infty}\left(\Gamma_{\mathrm{r}} \cup \Gamma_{0}\right)} & \leq 2 d \\
& \leq 2\left(k_{0}+2^{s /(s-1)}\left(c|\psi(0)|^{s-1}\right)^{1 / 2} \sqrt{\varrho f}\right) \\
& \leq c\left(k_{0}+\sqrt{\left\|f_{\Omega}\right\|_{L^{p_{1}(\Omega)}}^{2}+\left\|f_{\mathrm{r}}\right\|_{L^{p_{2}\left(\Gamma_{\mathrm{r}}\right)}}^{2}+\left\|f_{0}\right\|_{L^{p_{2}\left(\Gamma_{0}\right)}}^{2}}\right) \\
& \leq c_{0}\left(k_{0}+\left\|f_{\Omega}\right\|_{L^{p_{1}(\Omega)}}+\left\|f_{\mathrm{r}}\right\|_{\left.L^{p_{2}\left(\Gamma_{\mathrm{r}}\right)}+\left\|f_{0}\right\|_{L^{p_{2}\left(\Gamma_{0}\right)}}\right)}\right.
\end{aligned}
$$

by the equivalence of the Euclidian and the $L^{1}$-norm in $\mathbb{R}^{3}$.

REMARK 3.8. Due to the positivity of the trace operator (cf. Lemma 3.6), in Lemma 3.7, it would have sufficed to prove the boundedness of $y$ on $\Omega$. However, this would not have simplified the proof, as we still needed the direct estimates of the inhomogeneities on $\Gamma_{0}$ and $\Gamma_{\mathrm{r}}$ as carried out above.

4. The semilinear equation. Before we apply Lemma 3.7 to the semilinear equation in $(\mathrm{P})$, we will introduce the variational form of the state equation and recall a theorem of Laitinen and Tiihonen [10] that covers its solvability.

With the help of Lemma 3.3, Section 3.1, we are now able to derive the weak formulation of the state equation in $(\mathrm{P})$. To fix $G$, we specify $p$ in Lemma $3.3,(i)$, by $p=5 / 4$ 
and obtain $G: L^{5 / 4}\left(\Gamma_{\mathrm{r}}\right) \rightarrow L^{5 / 4}\left(\Gamma_{\mathrm{r}}\right)$. Then, formal integration by parts over $\Gamma_{0}$ and $\Gamma_{\mathrm{r}}$ yields

$$
\begin{aligned}
\int_{\Omega} \kappa \nabla y \cdot \nabla v d x+\int_{\Gamma_{\mathrm{r}}} G\left(\sigma|y|^{3} y\right) v d s+ & \int_{\Gamma_{0}} \varepsilon \sigma|y|^{3} y v d s \\
& =\int_{\Omega_{\mathrm{s}}} u v d x+\int_{\Gamma_{0}} \varepsilon \sigma y_{0}^{4} v d s \quad \forall v \in V,
\end{aligned}
$$

with $V=\left\{v \in H^{1}(\Omega) \mid \tau_{\mathrm{r}} v \in L^{5}\left(\Gamma_{\mathrm{r}}\right), \tau_{0} v \in L^{5}\left(\Gamma_{0}\right)\right\}$. Clearly, due to $G: L^{5 / 4}\left(\Gamma_{\mathrm{r}}\right) \rightarrow$ $L^{5 / 4}\left(\Gamma_{\mathrm{r}}\right)$, we have $G\left(\sigma|y|^{3} y\right) v \in L^{1}\left(\Gamma_{\mathrm{r}}\right)$ for all $y, v \in V$. The state space $V$ is equipped with the norm

$$
\|v\|_{V}=\|v\|_{H^{1}(\Omega)}+\|v\|_{L^{5}\left(\Gamma_{\mathrm{r}}\right)}+\|v\|_{L^{5}\left(\Gamma_{0}\right)} .
$$

A function $y \in V$ is said to be a weak solution of the state equation in (P), if (4.1) is fulfilled for every $v \in V$.

Theorem 4.1. [10, Theorem 2] For every $u \in H^{1}\left(\Omega_{\mathrm{s}}\right)^{*}$ and $y_{0} \in L^{5}\left(\Gamma_{0}\right)$, the semilinear equation (4.1) admits a unique solution in $V$.

The proof is mainly based on Brezis' theorem [16, Theorem 27.A] for pseudomonotone operators. Laitinen and Tiihonen showed in [10] that the semilinear differential operator defined by the left-hand side in (4.1) fulfills all assumptions of Brezis' theorem, i.e. it is pseudomonotone, bounded, and coercive on $V$. Thus, (4.1) admits at least one solution in $V$. The uniqueness then follows from a comparison principle [10, Theorem 4].

Next, we show the boundedness of solutions to (4.1). This result has been obtained by Tiihonen and Laitinen (see [10, Theorem 5]) in a slightly different setting by another method. Here, we need it for the nonlinear equation (4.1) as well as for its linearized version (see Section 6). In both cases, Lemma 3.7 can be applied.

TheOREM 4.2. Assume that $u \in L^{2}\left(\Omega_{\mathrm{s}}\right)$ and $y_{0} \in L^{16}\left(\Gamma_{0}\right)$. Then, there exists a constant $c_{0}$ only depending on $\Omega$ such that

$$
\|y\|_{L^{\infty}(\Omega)}+\|y\|_{L^{\infty}\left(\Gamma_{\mathrm{r}} \cup \Gamma_{0}\right)} \leq c_{0}\left(1+\|u\|_{L^{2}\left(\Omega_{\mathrm{s}}\right)}+\left\|y_{0}\right\|_{L^{16}\left(\Gamma_{0}\right)}^{4}\right)
$$

is valid.

Proof: To apply Lemma 3.7, we have to verify the Hypotheses (H1) and (H2). Here, we choose the state space $V \subset H^{1}(\Omega)$ for the space $W$. Since $y_{0} \in L^{16}\left(\Gamma_{0}\right)$ by assumption, we have $y_{0}^{4} \in L^{4}\left(\Gamma_{0}\right)$, and thus together with the assumed regularity of $u,(\mathrm{H} 1)$ is satisfied with $s=2$. To verify (H2), we show

$$
\int_{\Omega} \kappa \nabla y \cdot \nabla \varphi_{k} d x+\int_{\Gamma_{\mathrm{r}}} G\left(\sigma|y|^{3} y\right) \varphi_{k} d s+\int_{\Gamma_{0}} \varepsilon \sigma|y|^{3} y \varphi_{k} d s \geq c\left\|\varphi_{k}\right\|_{H^{1}(\Omega)}^{2}
$$

for each $k \geq 1$, which we will use in the estimate of the $\Gamma_{0}$-term. At first, we show the positivity of the radiation term on $\Gamma_{\mathrm{r}}$. To this aim, we split $\varphi_{k}=\varphi_{k}^{+}+\varphi_{k}^{-}$with

$$
\varphi_{k}^{+}(x):=\left\{\begin{array}{cl}
y(x)-k, & y(x) \geq k, \\
0, & y(x)<k,
\end{array} \text { and } \varphi_{k}^{-}(x):=\left\{\begin{array}{cc}
y(x)+k, & y(x) \leq-k, \\
0, & y(x)>-k .
\end{array}\right.\right.
$$


Hence, one obtains

$$
\int_{\Gamma_{\mathrm{r}}} G\left(\sigma|y|^{3} y\right) \varphi_{k} d s=\int_{\Gamma_{\mathrm{r}}} G\left(\sigma|y|^{3} y\right) \varphi_{k}^{+} d s+\int_{\Gamma_{\mathrm{r}}} G\left(\sigma|y|^{3} y\right) \varphi_{k}^{-} d s .
$$

Due to the linearity of $G$, the first integral on the right-hand side can be expressed by

$$
\int_{\Gamma_{\mathrm{r}}} G\left(\sigma|y|^{3} y\right) \varphi_{k}^{+} d s=\int_{\Gamma_{\mathrm{r}}} G(\sigma \Phi) \varphi_{k}^{+} d s+\int_{\Gamma_{\mathrm{r}}} G(\sigma \Psi) \varphi_{k}^{+} d s
$$

where

$$
\Phi(x):=\left\{\begin{array}{ll}
y(x)^{4}, y(x) \geq k, \\
k^{4}, y(x)<k,
\end{array} \quad \text { and } \quad \Psi(x):=\left\{\begin{array}{l}
0 \\
|y(x)|^{3} y(x)-k^{4}, y(x) \geq k \\
, y(x)<k .
\end{array}\right.\right.
$$

This partition is necessary since $G$ is nonlocal. Notice that $\Phi+\Psi=|y|^{3} y$ and $\Psi(x) \leq 0$ on $\Gamma_{\mathrm{r}}$. For the first addend in (4.5), we have, with $y_{k}(x):=\max \{y(x), k\}$,

$$
\int_{\Gamma_{\mathrm{r}}} G(\sigma \Phi) \varphi_{k}^{+} d s=\int_{\Gamma_{\mathrm{r}}} G\left(\sigma y_{k}^{4}\right)\left(y_{k}-k\right) d s=\int_{\Gamma_{\mathrm{r}}} G\left(\sigma y_{k}^{4}\right) y_{k} d s-\int_{\Gamma_{\mathrm{r}}} \sigma y_{k}^{4} G^{*} k d s \geq 0
$$

because of Lemma 3.3, (iii), and Lemma 3.4. Due to $G=I-H$ and the positivity of $H$ (see Lemma 3.3, $(i i)$ ), the second integral in (4.5) results in

$$
\int_{\Gamma_{\mathrm{r}}} G(\sigma \Psi) \varphi_{k}^{+} d s=\sigma \int_{\Gamma_{\mathrm{r}}} \Psi \varphi_{k}^{+} d s+\sigma \int_{\Gamma_{\mathrm{r}}} H(-\Psi) \varphi_{k}^{+} d s \geq 0
$$

since $\Psi(x) \neq 0$ implies $\varphi_{k}^{+}(x)=0$ and $-\Psi, \varphi_{k}^{+} \geq 0$ holds on $\Gamma_{\mathrm{r}}$.

Similarly, we prove the positivity of the second integral on the right-hand side of (4.4) with $y_{k}(x):=\min \{y(x),-k\}$, defining

$$
\Phi(x):=\left\{\begin{array}{ll}
|y(x)|^{3} y(x), & y(x) \leq-k, \\
-k^{4} & y(x)>-k,
\end{array} \quad \text { and } \quad \Psi(x):=\left\{\begin{array}{l}
0 \\
|y(x)|^{3} y(x)+k^{4}, y(x) \leq-k \\
y(x)>-k
\end{array}\right.\right.
$$

It remains to analyze the other integrals in (4.3). The first integral is estimated by

$$
\int_{\Omega} \kappa \nabla y \cdot \nabla \varphi_{k} d x \geq \kappa_{\min }\left\|\nabla \varphi_{k}\right\|_{L^{2}(\Omega)}^{2}
$$

since $\nabla \varphi_{k}(x)=0$ if $\nabla y(x) \neq \nabla \varphi_{k}(x)$. On $\Gamma_{0}$, using $k \geq k_{0}:=1$, we obtain

$$
\int_{\Gamma_{0}} \varepsilon \sigma|y|^{3} y \varphi_{k} d s \geq \varepsilon_{\min } \sigma k^{3}\left\|\varphi_{k}\right\|_{L^{2}\left(A_{0}(k)\right)}^{2} \geq \varepsilon_{\min } \sigma\left\|\varphi_{k}\right\|_{L^{2}\left(\Gamma_{0}\right)}^{2},
$$

as $y \varphi_{k}=\left(\operatorname{sign} \varphi_{k}\right)\left(\left|\varphi_{k}\right|+k\right) \varphi_{k} \geq \varphi_{k}^{2}$ is valid on $A_{0}(k)=\left\{x \in \Omega \mid \varphi_{k}(x) \neq 0\right\}$.

Due to the positivity of $\int_{\Gamma_{\mathrm{r}}} G\left(\sigma|y|^{3} y\right) \varphi_{k} d s$, we finally have

$$
\begin{aligned}
\int_{\Omega} \kappa \nabla y \cdot \nabla \varphi_{k} d x+\int_{\Gamma_{\mathrm{r}}} G\left(\sigma|y|^{3} y\right) \varphi_{k} d s+\int_{\Gamma_{0}} \varepsilon \sigma|y|^{3} y \varphi_{k} d s \\
\geq c\left(\left\|\nabla \varphi_{k}\right\|_{L^{2}(\Omega)}^{2}+\left\|\varphi_{k}\right\|_{L^{2}\left(\Gamma_{0}\right)}^{2}\right) \geq c\left\|\varphi_{k}\right\|_{H^{1}(\Omega)}^{2} .
\end{aligned}
$$


Therefore, we can apply Lemma 3.7, and estimate (3.6) gives with $s=2, k_{0}=1$, $f_{\Omega}=u$, and $f_{0}=y_{0}^{4}$

$$
\begin{aligned}
\|y\|_{L^{\infty}(\Omega)}+\|y\|_{L^{\infty}\left(\Gamma_{\mathrm{r}} \cup \Gamma_{0}\right)} & \leq c_{0}\left(1+\|u\|_{L^{2}\left(\Omega_{\mathrm{s}}\right)}+\left\|y_{0}^{4}\right\|_{L^{4}\left(\Gamma_{0}\right)}\right) \\
& \leq c_{0}\left(1+\|u\|_{L^{2}\left(\Omega_{\mathrm{s}}\right)}+\left\|y_{0}\right\|_{L^{16}\left(\Gamma_{0}\right)}^{4}\right) .
\end{aligned}
$$

For the discussion of the linearized equation, see Section 6, we need another property of the semilinear solution, namely the following maximum principle:

TheOREM 4.3. Suppose that $u(x) \geq 0$ a.e. in $\Omega_{\mathrm{s}}$ and $y_{0}(x) \geq \vartheta>0$ a.e. on $\Gamma_{0}$. If $y$ is the solution of (4.1), then $y(x) \geq \vartheta$ holds a.e. on $\Omega$ and a.e. on $\Gamma_{\mathrm{r}} \cup \Gamma_{0}$.

Proof: This time, we use the following test function in (4.1):

$$
\rho_{\vartheta}(x):= \begin{cases}y(x)-\vartheta & , y(x) \leq \vartheta \\ 0 & , y(x)>\vartheta\end{cases}
$$

Furthermore, we define $\Omega^{-}=\{x \in \Omega \mid y(x) \leq \vartheta\}$ and introduce analogous definitions for $\Omega_{\mathrm{s}}^{-}, \Gamma_{0}^{-}$and $\Gamma_{\mathrm{r}}^{-}$. Thus, we have $\left.\rho_{\vartheta}\right|_{\Omega \backslash \Omega^{-}}=0$, and (4.1) reads

$$
\begin{aligned}
\int_{\Omega^{-}} \kappa \nabla y \cdot \nabla \rho_{\vartheta} d x+ & \int_{\Gamma_{\mathrm{r}}} G\left(\sigma|y|^{3} y\right) \rho_{\vartheta} d s \\
& +\int_{\Gamma_{0}^{-}} \varepsilon \sigma\left(|y|^{3} y-y_{0}^{4}\right) \rho_{\vartheta} d s=\int_{\Omega_{\mathrm{s}}^{-}} u \rho_{\vartheta} d x .
\end{aligned}
$$

Next, as in the proof of Theorem 4.2, one can show the positivity of the integral over $\Gamma_{\mathrm{r}}$ by decomposition:

$$
\int_{\Gamma_{\mathrm{r}}} G\left(\sigma|y|^{3} y\right) \rho_{\vartheta} d s=\int_{\Gamma_{\mathrm{r}}} G(\sigma \Phi) \rho_{\vartheta} d s+\int_{\Gamma_{\mathrm{r}}} G(\sigma \Psi) \rho_{\vartheta} d s
$$

with

$$
\Phi(x)=\left\{\begin{array}{ll}
|y(x)|^{3} y(x), y(x) \leq \vartheta, \\
\vartheta^{4}, y(x)>\vartheta,
\end{array} \quad \text { and } \quad \Psi(x)= \begin{cases}0 & , y(x) \leq \vartheta \\
|y(x)|^{3} y(x)-\vartheta^{4}, & y(x)>\vartheta\end{cases}\right.
$$

Therefore, (4.6) results in

$$
\int_{\Gamma_{0}^{-}} \varepsilon \sigma\left(y_{0}^{4}-|y|^{3} y\right) \rho_{\vartheta} d s \geq \int_{\Omega^{-}} \kappa \nabla y \cdot \nabla \rho_{\vartheta} d x-\int_{\Omega_{\mathrm{s}}^{-}} u \rho_{\vartheta} d x \geq 0
$$

since $\nabla \rho_{\vartheta}=\nabla y$ on $\Omega^{-}, \rho_{\vartheta} \leq 0$ by definition, and $u \geq 0$ by assumption. On $\Gamma_{0}^{-}$, we also have $y-\vartheta \leq 0$ implying $y_{0}^{4}-|y|^{3} y \geq 0$. Thus, we obtain

$$
0 \leq \int_{\Gamma_{0}^{-}} \varepsilon \sigma\left(y_{0}^{4}-|y|^{3} y\right) \rho_{\vartheta} d s=\int_{\Gamma_{0}^{-}} \varepsilon \sigma\left(y_{0}^{4}-|y|^{3} y\right)(y-\vartheta) d s \leq 0,
$$

and, consequently, $\left|\Gamma_{0}^{-}\right|=0$ or $y=y_{0}$ or $y=\vartheta$ a.e. on $\Gamma_{0}$, which, in each case, yields the assertion on $\Gamma_{0}$. Therefore, we have $\rho_{\vartheta} \in H_{0}^{1}(\Omega)$. 
Now, from (4.7), it follows that

$$
\kappa_{\min }\left\|\nabla \rho_{\vartheta}\right\|_{L^{2}(\Omega)} \leq \int_{\Gamma_{0}^{-}} \varepsilon \sigma\left(y_{0}^{4}-|y|^{3} y\right) \rho_{\vartheta} d s+\int_{\Omega_{\mathrm{s}}^{-}} u \rho_{\vartheta} d x \leq 0,
$$

since $\nabla \rho_{\vartheta}=0$ in $\Omega \backslash \Omega^{-}$. Hence $\left\|\nabla \rho_{\vartheta}\right\|_{L^{2}(\Omega)}=\left\|\rho_{\vartheta}\right\|_{H_{0}^{1}(\Omega)}=0$ (notice that we have already shown $\rho_{\vartheta}=0$ a.e. on $\Gamma_{0}$ ). Thus $\rho_{\vartheta}=0$ a.e. in $\Omega$. Thus, we have shown $y \geq \vartheta$ a.e. on both $\Omega$ and $\Gamma_{0}$, and Lemma 3.6 then ensures $y \geq \vartheta$ a.e. on $\Gamma_{\mathrm{r}}$.

5. Existence of an optimal solution. With the results of Section 4 at hand, the proof of existence of an optimal solution for the optimal control problem $(\mathrm{P})$ is rather standard. We start with the following lemma:

LEMMA 5.1. Let $u \in L^{6 / 5}\left(\Omega_{\mathrm{s}}\right), y_{0} \in L^{16 / 3}\left(\Gamma_{0}\right)$ be given and assume that the hypotheses of Theorem 4.3 are fulfilled. Then a constant $c_{1}$ exists only depending on $\Omega$ with

$$
\|y\|_{H^{1}(\Omega)} \leq c_{1}\left(\|u\|_{L^{6 / 5}\left(\Omega_{\mathrm{s}}\right)}+\left\|y_{0}\right\|_{L^{16 / 3}\left(\Gamma_{0}\right)}^{4}\right)
$$

Proof: Using the solution $y$ as a test function in (4.1) yields

$$
\int_{\Omega} \kappa|\nabla y|^{2} d x+\int_{\Gamma_{\mathrm{r}}} G\left(\sigma|y|^{3} y\right) y d s+\int_{\Gamma_{0}} \varepsilon \sigma|y|^{3} y^{2} d s=\int_{\Omega_{\mathrm{s}}} u y d x+\int_{\Gamma_{0}} \varepsilon \sigma y_{0}^{4} y d s .
$$

Owing to Lemma 3.3, (iii), and the maximum principle in Theorem 4.3, we have

$$
\begin{aligned}
& \int_{\Omega} \kappa|\nabla y|^{2} d x+\int_{\Gamma_{\mathrm{r}}} G\left(\sigma|y|^{3} y\right) y d s+\int_{\Gamma_{0}} \varepsilon \sigma|y|^{3} y^{2} d s \\
& \geq \kappa_{\min }\|\nabla y\|_{L^{2}(\Omega)}+\varepsilon_{\min } \sigma \vartheta^{3}\|y\|_{L^{2}\left(\Gamma_{0}\right)} \geq c\|y\|_{H^{1}(\Omega)}^{2} .
\end{aligned}
$$

Therefore, by trace and embedding theorems,

$$
\begin{aligned}
\|y\|_{H^{1}(\Omega)}^{2} & \leq c\left(\int_{\Omega_{\mathrm{s}}} u y d x+\int_{\Gamma_{0}} \varepsilon \sigma y_{0}^{4} y d s\right) \\
& \leq c\left(\|u\|_{L^{6 / 5}\left(\Omega_{\mathrm{s}}\right)}\|y\|_{L^{6}\left(\Omega_{\mathrm{s}}\right)}+\left\|y_{0}^{4}\right\|_{L^{4 / 3}\left(\Gamma_{0}\right)}\|y\|_{L^{4}\left(\Gamma_{0}\right)}\right) \\
& \leq c_{1}\left(\|u\|_{L^{6 / 5}\left(\Omega_{\mathrm{s}}\right)}+\left\|y_{0}\right\|_{L^{16 / 3}\left(\Gamma_{0}\right)}^{4}\right)\|y\|_{H^{1}(\Omega)},
\end{aligned}
$$

which establishes the case.

TheOREM 5.2. Assume that $u_{a} \geq 0, y_{0} \in L^{16}\left(\Gamma_{0}\right)$, and $y_{0} \geq \vartheta>0$. Then there exists a solution $(\bar{u}, \bar{y}) \in L^{\infty}\left(\Omega_{\mathrm{s}}\right) \times V^{\infty}$ to $(\mathrm{P})$.

Proof: As mentioned above, the proof follows standard arguments. We start with a sequence $\left\{\left(u_{n}, y_{n}\right)\right\}_{n=1}^{\infty}$ converging to the infimum $\bar{J} \geq 0$ of the objective functional in $(\mathrm{P})$, i.e. $J\left(u_{n}, y_{n}\right) \rightarrow \bar{J}$. We will now show the convergence of a subsequence of $\left\{\left(u_{n}, y_{n}\right)\right\}_{n=1}^{\infty}$ to an optimal solution $(\bar{u}, \bar{y})$.

The box constraints ensure that $u$ is bounded in $L^{2}\left(\Omega_{\mathrm{s}}\right)$. Thus, we can select a weakly converging subsequence, w.l.o.g. again denoted by $u_{n}, u_{n} \rightarrow \bar{u}$. Since $U_{\text {ad }}$ is a closed 
and convex subset of $L^{2}\left(\Omega_{\mathrm{s}}\right)$, we have $\bar{u} \in U_{\mathrm{ad}}$.

In addition to the boundedness, the $u_{n}$ are also nonnegative because of $u_{a} \geq 0$. Together with the assumptions on $y_{0}$, this yields the boundedness of $\left\|y_{n}\right\|_{H^{1}(\Omega)}$ thanks to Lemma 5.1. Hence, we can select a weakly converging subsequence and w.l.o.g. $y_{n}$ itself, i.e. $y_{n} \rightarrow \bar{y}, n \rightarrow \infty$, in $H^{1}(\Omega)$. The trace theorem and the compact embedding of $H^{1 / 2}\left(\Gamma_{\mathrm{r}} \cup \Gamma_{0}\right)$ in $L^{2}\left(\Gamma_{\mathrm{r}} \cup \Gamma_{0}\right)$ then give

$$
\tau y_{n} \rightarrow \tau \bar{y}, n \rightarrow \infty \quad \text { in } L^{2}\left(\Gamma_{\mathrm{r}} \cup \Gamma_{0}\right) .
$$

Now, the convergence of the nonlinearities can be derived on $\Gamma_{\mathrm{r}}$ and $\Gamma_{0}$. Since $u_{n}$, $n \in \mathbb{N}$, is uniformly bounded in $L^{2}\left(\Omega_{\mathrm{s}}\right)$, and $y_{0} \in L^{16}\left(\Gamma_{0}\right)$ by assumption, Theorem 4.2 ensures that a constant $d$ exists with $|y(x)| \leq d$ a.e. in $\Omega$ and a.e. in $\Gamma_{\mathrm{r}} \cup \Gamma_{0}$. One can easily verify that the Nemytskii operator $\Phi(y):=|y|^{3} y$ satisfies

$$
\left\|\Phi\left(y_{n}\right)-\Phi\left(y_{m}\right)\right\|_{L^{2}\left(\Gamma_{\mathrm{r}} \cup \Gamma_{0}\right)} \leq L(d)\left\|y_{n}-y_{m}\right\|_{L^{2}\left(\Gamma_{\mathrm{r}} \cup \Gamma_{0}\right)}
$$

for all $y_{n}, y_{m} \in\left\{y \in L^{\infty}\left(\Gamma_{0} \cup \Gamma_{\mathrm{r}}\right)|| y(x) \mid \leq d\right.$ a.e. on $\left.\Gamma_{0} \cup \Gamma_{\mathrm{r}}\right\}$. Since this set is closed in $L^{2}\left(\Gamma_{\mathrm{r}} \cup \Gamma_{0}\right)$, also $|\bar{y}(x)| \leq d$ holds true, and the convergence in $L^{2}\left(\Gamma_{\mathrm{r}} \cup \Gamma_{0}\right)$ together with (5.1) yields

$$
\left|\tau y_{n}\right|^{3} \tau y_{n} \rightarrow|\tau \bar{y}|^{3} \tau \bar{y} \quad \text { in } L^{2}\left(\Gamma_{\mathrm{r}} \cup \Gamma_{0}\right)
$$

Consider now the variational equation (4.1) when passing to the limit. For $\left(u_{n}, y_{n}\right)$, it reads

$$
\begin{aligned}
\int_{\Omega} \kappa \nabla y_{n} \cdot \nabla v d x+\int_{\Gamma_{\mathrm{r}}} G\left(\sigma\left|y_{n}\right|^{3} y_{n}\right) v d s+ & \int_{\Gamma_{0}} \varepsilon \sigma\left|y_{n}\right|^{3} y_{n} v d s= \\
& \int_{\Omega_{\mathrm{s}}} u_{n} v d x+\int_{\Gamma_{0}} \varepsilon \sigma y_{0}^{4} v d s .
\end{aligned}
$$

Due to $y_{n} \rightarrow \bar{y}$ in $H^{1}(\Omega)$, the first integral on the left-hand side converges to $\int_{\Omega} \kappa \nabla \bar{y}$. $\nabla v d x$. The boundary integrals on the left-hand side converge because of (5.2) and the continuity of $G$. Finally, the inhomogeneity converges owing to $u_{n}-\bar{u}$ in $L^{2}\left(\Omega_{\mathrm{s}}\right)$. Therefore, the limit $(\bar{u}, \bar{y})$ satisfies the weak formulation (4.1).

The optimality of $(\bar{y}, \bar{u})$ follows in a standard way by the lower semicontinuity of $J$

6. The linearized equation. In this section, we investigate the linearization of the state equation (4.1) at a fixed reference pair $(\bar{y}, \bar{u}) \in V^{\infty} \times L^{2}\left(\Omega_{\mathrm{s}}\right)^{1}$. The linearized equation arises from the differentiation of the solution operator associated with the semilinear state equation $S: L^{2}\left(\Omega_{\mathrm{s}}\right) \rightarrow V^{\infty}$ mapping $u$ to $y$. This derivative of $S$ at $(\bar{y}, \bar{u})$ appears in the variational inequality (see Section 7 ). More precisely, we consider the linearized equation with a more general inhomogeneity, i.e. in the form

$$
\begin{aligned}
-\operatorname{div}(\kappa \nabla y) & =f_{\Omega} & & \text { in } \Omega \\
\kappa_{\mathrm{s}}\left(\frac{\partial y}{\partial n_{\mathrm{r}}}\right)_{\mathrm{s}}-\kappa_{\mathrm{g}}\left(\frac{\partial y}{\partial n_{\mathrm{r}}}\right)_{\mathrm{g}}+4 G\left(\sigma|\bar{y}|^{3} y\right) & =f_{\mathrm{r}} & & \text { on } \Gamma_{\mathrm{r}} \\
\kappa_{\mathrm{s}} \frac{\partial y}{\partial n_{0}}+4 \varepsilon \sigma|\bar{y}|^{3} y & =f_{0} & & \text { on } \Gamma_{0}
\end{aligned}
$$

\footnotetext{
${ }^{1}$ Here and in the following, we will denote a fixed solution of the semilinear equation as well as an optimal solution by $(\bar{u}, \bar{y})$.
} 
with $\bar{y}=S \bar{u} \in V^{\infty}$ and arbitrary functions $\left(f_{\Omega}, f_{\mathrm{r}}, f_{0}\right)$ in $L^{2}(\Omega) \times L^{2}\left(\Gamma_{\mathrm{r}}\right) \times L^{2}\left(\Gamma_{0}\right)$. In the next section, we will show that $S$ is indeed Fréchet differentiable and that its derivative $y=S^{\prime}(\bar{u}) u$ corresponds to the solution of (6.1) with $f_{\Omega}=u, f_{\mathrm{r}}=0$, and $f_{0}=0$. However, we first focus on (6.1), and, in the present section, prove the existence of a unique solution in $V^{\infty}$. The existence theory is based on the theory of Fredholm operators and has to account for eigenvalues. This is due to the fact that the Lax-Milgram lemma cannot directly be applied because of the lack of coercivity, caused by the radiation operator $G$ in the linearized case. The boundedness of the solution is again shown by Lemma 3.7.

The variational equation of (6.1) reads

$$
\begin{aligned}
& \int_{\Omega} \kappa \nabla y \cdot \nabla v d x+\int_{\Gamma_{0}} 4 \varepsilon \sigma|\bar{y}|^{3} y v d s \\
&=\int_{\Omega} f_{\Omega} v d x+\int_{\Gamma_{\mathrm{r}}}\left(f_{\mathrm{r}}-4 G\left(\sigma|\bar{y}|^{3} y\right)\right) v d s+\int_{\Gamma_{0}} f_{0} v d s \quad \forall v \in H^{1}(\Omega) .
\end{aligned}
$$

In view of $\bar{y} \in V^{\infty}$ and $\bar{y} \geq \vartheta>0$ (Theorem 4.3), the bilinear form defined by the left-hand side of (6.2) is bounded and coercive in $H^{1}(\Omega)$. Therefore, the Lax-Milgram lemma yields continuous linear operators $B_{\Omega}: L^{2}(\Omega) \rightarrow H^{1}(\Omega), B_{\mathrm{r}}: L^{2}\left(\Gamma_{\mathrm{r}}\right) \rightarrow H^{1}(\Omega)$, and $B_{0}: L^{2}\left(\Gamma_{0}\right) \rightarrow H^{1}(\Omega)$ such that, with $\tilde{y}:=\tau_{\mathrm{r}} y$ :

$$
y=B_{\Omega} f_{\Omega}+B_{\mathrm{r}}\left(f_{\mathrm{r}}-4 G\left(\sigma\left|\tau_{\mathrm{r}} \bar{y}\right|^{3} \tilde{y}\right)\right)+B_{0} f_{0}
$$

We are now in a position to formulate the existence theorem for (6.1):

TheOREM 6.1. Assume $\bar{y} \in V^{\infty}, \bar{y} \geq \vartheta>0$, and that $\lambda=1$ is not an eigenvalue of $B(\bar{y})(\cdot):=-\tau_{\mathrm{r}} B_{\mathrm{r}}\left(4 G\left(\sigma\left|\tau_{\mathrm{r}} \bar{y}\right|^{3} \cdot\right)\right.$ with $B(\bar{y}): L^{2}\left(\Gamma_{\mathrm{r}}\right) \rightarrow L^{2}\left(\Gamma_{\mathrm{r}}\right)$. Then, to every $\left(f_{\Omega}, f_{\mathrm{r}}, f_{0}\right) \in L^{2}(\Omega) \times L^{2}\left(\Gamma_{\mathrm{r}}\right) \times L^{2}\left(\Gamma_{0}\right)$, there exists a unique solution $y$ of $(6.1)$ in $H^{1}(\Omega)$.

Proof: In the following, we will suppress the dependency of $B$ on $\bar{y}$, since $\bar{y}$ is fixed. Thus, we simply write $B$ instead of $B(\bar{y})$. Applying the trace operator $\tau_{\mathrm{r}}$ to both sides, (6.3) it results in

$$
(I-B) \tilde{y}=\tilde{y}+\tau_{\mathrm{r}} B_{\mathrm{r}}\left(4 G\left(\sigma\left|\tau_{\mathrm{r}} \bar{y}\right|^{3} \tilde{y}\right)\right)=\tau_{\mathrm{r}}\left(B_{\Omega} f_{\Omega}+B_{\mathrm{r}} f_{\mathrm{r}}+B_{0} f_{0}\right) .
$$

Since $B_{\mathrm{r}}: L^{2}\left(\Gamma_{\mathrm{r}}\right) \rightarrow H^{1}(\Omega)$, and $\bar{y} \in V^{\infty}$ by assumption, and, thus, $\tau_{\mathrm{r}} \bar{y} \in L^{\infty}\left(\Gamma_{\mathrm{r}}\right)$, it follows that $B$ maps all $\tilde{y} \in L^{2}\left(\Gamma_{\mathrm{r}}\right)$ to $H^{1 / 2}\left(\Gamma_{\mathrm{r}}\right)$. Due to the compact embedding, $B$ is a compact operator from $L^{2}\left(\Gamma_{\mathrm{r}}\right)$ to $L^{2}\left(\Gamma_{\mathrm{r}}\right)$. Therefore, $\lambda=1$ is either one of the countably many eigenvalues of $B$, or $(I-B)$ is continuously invertible. Thus, we obtain a unique solution $\tilde{y} \in L^{2}\left(\Gamma_{\mathrm{r}}\right)$ of (6.4) prescribed that $\lambda=1$ is not an eigenvalue of $B$.

Furthermore, for every $\tilde{y} \in L^{2}\left(\Gamma_{\mathrm{r}}\right)$, we have $G\left(\sigma\left|\tau_{\mathrm{r}} \bar{y}\right|^{3} \tilde{y}\right) \in L^{2}\left(\Gamma_{\mathrm{r}}\right)$ by means of $\bar{y} \in V^{\infty}$, and, hence, (6.3) admits a unique solution in $H^{1}(\Omega)$.

ThEOREM 6.2. Suppose that the assumptions of Theorem 6.1 hold true. Then, for all $f_{\Omega} \in L^{2}(\Omega), f_{\mathrm{r}} \in L^{4}\left(\Gamma_{\mathrm{r}}\right)$, and $f_{0} \in L^{4}\left(\Gamma_{\mathrm{r}}\right)$, there exists a constant $c_{2}$ only depending on $\Omega$ such that

$$
\|y\|_{L^{\infty}(\Omega)}+\|y\|_{L^{\infty}\left(\Gamma_{\mathrm{r}} \cup \Gamma_{0}\right)} \leq c_{2}\left(\left\|f_{\Omega}\right\|_{L^{2}(\Omega)}+\left\|f_{\mathrm{r}}\right\|_{L^{4}\left(\Gamma_{\mathrm{r}}\right)}+\left\|f_{0}\right\|_{L^{4}\left(\Gamma_{0}\right)}\right)
$$


holds true.

Proof: Again, we use Lemma 3.7 to prove the boundedness, this time with $W=$ $H^{1}(\Omega)$. We apply it to $(6.2)$ for $F$ defined by

$$
\langle F(y), v\rangle=\int_{\Omega} \kappa \nabla y \cdot \nabla v d x+\int_{\Gamma_{0}} 4 \varepsilon \sigma|\bar{y}|^{3} y v d s .
$$

Hypothesis (H2) clearly holds with $k_{0}=0$, owing to the coercivity of the bilinear form.

Now, Hypothesis (H1), i.e. the regularity of the right-hand side is the critical point, since the inhomogeneity on $\Gamma_{\mathrm{r}}$ in (6.2) depends on the solution $y$. As before, we choose $s=2$ in (H1), and, thus, the required regularities coincide with the assumptions on $f_{\Omega}, f_{\mathrm{r}}$, and $f_{0}$.

The part of the inhomogeneity depending on $y$ and $\bar{y}$ is given by $-4 G\left(\sigma|\bar{y}|^{3} y\right)$. It belongs to $L^{4}\left(\Gamma_{\mathrm{r}}\right)$, since $y \in H^{1}(\Omega)$, and, therefore, $\tau_{\mathrm{r}} y \in L^{4}\left(\Gamma_{\mathrm{r}}\right)$ in addition to $\bar{y} \in V^{\infty}$. Consequently, also (H1) is satisfied, and Lemma 3.7 can be applied.

It remains to verify that a bound exists that is independent of $y$. With $k_{0}=0$, estimate (3.6) on page 6 gives

$$
\begin{aligned}
\|y\|_{L^{\infty}(\Omega)} & +\|y\|_{L^{\infty}\left(\Gamma_{\mathrm{r}} \cup \Gamma_{0}\right)} \\
& \leq c_{0}\left(\left\|f_{\Omega}\right\|_{L^{2}(\Omega)}+\left\|f_{\mathrm{r}}\right\|_{L^{4}\left(\Gamma_{\mathrm{r}}\right)}+\left\|4 G\left(\sigma|\bar{y}|^{3} y\right)\right\|_{L^{4}\left(\Gamma_{\mathrm{r}}\right)}+\left\|f_{0}\right\|_{L^{4}\left(\Gamma_{0}\right)}\right) .
\end{aligned}
$$

We estimate the term with $y$ by

$$
\left\|G\left(\sigma|\bar{y}|^{3} y\right)\right\|_{L^{4}\left(\Gamma_{\mathrm{r}}\right)} \leq c\|G\|_{\mathcal{L}\left(L^{4}\left(\Gamma_{\mathrm{r}}\right)\right)}\|\bar{y}\|_{L^{\infty}\left(\Gamma_{\mathrm{r}}\right)}^{3}\|y\|_{H^{1}(\Omega)} .
$$

With (6.3) and the continuity of $B_{\Omega}, B_{\mathrm{r}}$, and $B_{0}$, we obtain because of the boundedness of $\bar{y}$ :

$$
\begin{aligned}
\|y\|_{H^{1}(\Omega)} \leq & \left\|B_{\Omega} f_{\Omega}+B_{\mathrm{r}} f_{\mathrm{r}}+B_{0} f_{0}\right\|_{H^{1}(\Omega)} \\
& +c\left\|B_{\mathrm{r}}\right\|_{\mathcal{L}\left(L^{2}\left(\Gamma_{\mathrm{r}}\right), H^{1}(\Omega)\right)}\|G\|_{\mathcal{L}\left(L^{2}\left(\Gamma_{\mathrm{r}}\right)\right)}\|\bar{y}\|_{L^{\infty}\left(\Gamma_{\mathrm{r}}\right)}^{3}\|\tilde{y}\|_{L^{2}\left(\Gamma_{\mathrm{r}}\right)} \\
\leq & c\left(\left\|f_{\Omega}\right\|_{L^{2}(\Omega)}+\left\|f_{\mathrm{r}}\right\|_{L^{4}\left(\Gamma_{\mathrm{r}}\right)}+\left\|f_{0}\right\|_{L^{4}\left(\Gamma_{0}\right)}+\|\tilde{y}\|_{L^{2}\left(\Gamma_{\mathrm{r}}\right)}\right) .
\end{aligned}
$$

Due to $(6.4),\|\tilde{y}\|_{L^{2}\left(\Gamma_{\mathrm{r}}\right)}$ is bounded by

$$
\begin{aligned}
\|\tilde{y}\|_{L^{2}\left(\Gamma_{\mathrm{r}}\right)} & \leq\left\|(I-B)^{-1}\right\|_{\mathcal{L}\left(L^{2}\left(\Gamma_{\mathrm{r}}\right)\right)} \cdot\left\|B_{\Omega} f_{\Omega}+B_{\mathrm{r}} f_{\mathrm{r}}+B_{0} f_{0}\right\|_{L^{2}\left(\Gamma_{\mathrm{r}}\right)} \\
& \leq c\left(\left\|f_{\Omega}\right\|_{L^{2}(\Omega)}+\left\|f_{\mathrm{r}}\right\|_{L^{4}\left(\Gamma_{\mathrm{r}}\right)}+\left\|f_{0}\right\|_{L^{4}\left(\Gamma_{0}\right)}\right)
\end{aligned}
$$

where Fredholm theory grants $\left\|(I-B)^{-1}\right\|_{\mathcal{L}\left(L^{2}\left(\Gamma_{\mathrm{r}}\right)\right)}<\infty$. Combing $(6.7)-(6.9)$ yields

$$
\left\|G\left(\sigma|\bar{y}|^{3} y\right)\right\|_{L^{4}\left(\Gamma_{\mathrm{r}}\right)} \leq \tilde{c}\left(\left\|f_{\Omega}\right\|_{L^{2}(\Omega)}+\left\|f_{\mathrm{r}}\right\|_{L^{4}\left(\Gamma_{\mathrm{r}}\right)}+\left\|f_{0}\right\|_{L^{4}\left(\Gamma_{0}\right)}\right)
$$

with a constant $\tilde{c}$ only depending on $\Omega$. Inserting (6.10) in (6.6) finally gives

$$
\begin{aligned}
\|y\|_{L^{\infty}(\Omega)}+\|y\|_{L^{\infty}\left(\Gamma_{\mathrm{r}} \cup \Gamma_{0}\right)} & \leq c_{0}(1+\tilde{c})\left(\left\|f_{\Omega}\right\|_{L^{2}(\Omega)}+\left\|f_{\mathrm{r}}\right\|_{L^{4}\left(\Gamma_{\mathrm{r}}\right)}+\left\|f_{0}\right\|_{L^{4}\left(\Gamma_{0}\right)}\right) \\
& \leq c_{2}\left(\left\|f_{\Omega}\right\|_{L^{2}(\Omega)}+\left\|f_{\mathrm{r}}\right\|_{L^{4}\left(\Gamma_{\mathrm{r}}\right)}+\left\|f_{0}\right\|_{L^{4}\left(\Gamma_{0}\right)}\right) .
\end{aligned}
$$


7. First order necessary optimality conditions. With the results of Sections 4 and 6 , we are now able to show the Fréchet differentiability of the semilinear PDE solution operator $S: u \mapsto y$ from $L^{2}\left(\Omega_{\mathrm{s}}\right)$ to $V^{\infty}$, see Sec. 7.1. Defining $J(y, u)=$ $J(S u, u)=: j(u)$, a standard argument then yields the variational inequality for the optimal pair $(\bar{u}, \bar{y})$ :

$$
j^{\prime}(\bar{u})(u-\bar{u})=(\nabla \bar{y}-z, \nabla y)_{L^{2}\left(\Omega_{\mathrm{g}}\right)}+\nu(\bar{u},(u-\bar{u}))_{L^{2}\left(\Omega_{\mathrm{s}}\right)} \geq 0 \quad \forall u \in U_{\mathrm{ad}},
$$

with $\bar{y}=S \bar{u}$ and $y=S^{\prime}(\bar{u})(u-\bar{u})$. The latter relation means that $y$ satisfies the PDE

$$
\begin{aligned}
-\operatorname{div}\left(\kappa_{\mathrm{s}} \nabla y\right) & =u-\bar{u} & & \text { in } \Omega_{\mathrm{s}}, \\
-\operatorname{div}\left(\kappa_{\mathrm{g}} \nabla y\right) & =0 & & \text { in } \Omega_{\mathrm{g}}, \\
\kappa_{\mathrm{S}}\left(\frac{\partial y}{\partial n_{r}}\right)_{\mathrm{s}}-\kappa_{\mathrm{g}}\left(\frac{\partial y}{\partial n_{r}}\right)_{\mathrm{g}}+4 G\left(\sigma|\bar{y}|^{3} y\right) & =0 & & \text { on } \Gamma_{\mathrm{r}}, \\
\kappa_{\mathrm{s}} \frac{\partial y}{\partial n_{0}}+4 \varepsilon \sigma|\bar{y}|^{3} y & =0 & & \text { on } \Gamma_{0} .
\end{aligned}
$$

Note that (7.2) constitutes a special case of (6.1).

In Section 7.2, we transform the variational inequality (7.1) into the standard projection formula depending on the adjoint state $p$.

7.1. Differentiability of the control-to-state operator. We show the differentiability of $S$ by the implicit function theorem. To that end, let us introduce an auxiliary operator $T$, such that $S$ is implicitly defined by $T(u, S(u))=0$. Preparing the definition of $T$, we consider the following equation that is equivalent to the weak formulation of the semilinear PDE (4.1):

$$
\begin{aligned}
\int_{\Omega} \kappa \nabla y & \cdot \nabla v d x+\int_{\Gamma_{0}} \lambda y v d s \\
& =-\int_{\Gamma_{\mathrm{r}}} G\left(\sigma|y|^{3} y\right) v d s+\int_{\Gamma_{0}}\left(\lambda y+\varepsilon \sigma y_{0}^{4}-\varepsilon \sigma|y|^{3} y\right) v d s+\int_{\Omega_{\mathrm{s}}} u v d x
\end{aligned}
$$

with a fixed $\lambda>0$. Due to the positivity of $\lambda$, the left-hand side in (7.3) represents a bounded, coercive bilinear form in $H^{1}(\Omega)$. Thus, for every right-hand side in $H^{1}(\Omega)^{*}$, we have a unique solution in $H^{1}(\Omega)$. Furthermore, similar to the proof of Theorem 6.2 , it follows that this solution is bounded if the right-hand side is sufficiently regular, i.e. in $L^{2}(\Omega) \times L^{4}\left(\Gamma_{\mathrm{r}}\right) \times L^{4}\left(\Gamma_{0}\right)$. Thus, continuous operators $\tilde{B}_{\Omega_{\mathrm{s}}}: L^{2}\left(\Omega_{\mathrm{S}}\right) \rightarrow V^{\infty}$, $\tilde{B}_{0}: L^{4}\left(\Gamma_{0}\right) \rightarrow V^{\infty}$, and $\tilde{B}_{\mathrm{r}}: L^{4}\left(\Gamma_{\mathrm{r}}\right) \rightarrow V^{\infty}$ exist with

$$
y=\tilde{B}_{\Omega_{\mathrm{s}}} u-\tilde{B}_{\mathrm{r}}\left(G\left(\sigma|y|^{3} y\right)\right)+\tilde{B}_{0}\left(\lambda y+\varepsilon \sigma y_{0}^{4}-\varepsilon \sigma|y|^{3} y\right),
$$

and our auxiliary operator is given by

$$
T(u, y):=y-\tilde{B}_{\Omega_{\mathrm{s}}} u+\tilde{B}_{\mathrm{r}}\left(G\left(\sigma|y|^{3} y\right)\right)-\tilde{B}_{0}\left(\lambda y+\varepsilon \sigma y_{0}^{4}-\varepsilon \sigma|y|^{3} y\right),
$$

with $T: L^{2}\left(\Omega_{\mathrm{s}}\right) \times V^{\infty} \rightarrow V^{\infty}$.

THEOREM 7.1. Assume that $u_{a} \geq 0, y_{0} \in L^{16}\left(\Gamma_{0}\right), y_{0} \geq \vartheta>0$ and $\lambda=1$ is not an eigenvalue of $B$, where $B=B(\bar{y})$ is as defined in Theorem 6.1. Then, $S: L^{2}\left(\Omega_{\mathrm{s}}\right) \rightarrow$ 
$V^{\infty}$ is Fréchet differentiable at $(\bar{u}, \bar{y})$, and $y:=S^{\prime}(\bar{u})(u-\bar{u})$ is given by the solution of the linearized equation (7.2).

Proof: According to the definition of $T$ in (7.4) and because of Theorems 4.1 and 4.2, $y=S(u)$ if, and only if, $T(u, y)=T(u, S u)=0$, since this equation corresponds to the semilinear equation (4.1). To prove the differentiability of $S$, it thus suffices to verify the hypotheses of the implicit function theorem for $T$.

The Nemytskii operator $\Phi(y)=|y|^{3} y$ is continuously Fréchet differentiable from $L^{\infty}\left(\Gamma_{\mathrm{r}} \cup \Gamma_{0}\right)$ to $L^{\infty}\left(\Gamma_{\mathrm{r}} \cup \Gamma_{0}\right)$, see [9]. The other operators in the definition of $T$ are all continuous and linear operators, and, thus, trivially continuously Fréchet differentiable on their particular spaces, i.e. $G$ from $L^{\infty}\left(\Gamma_{\mathrm{r}}\right)$ to $L^{\infty}\left(\Gamma_{\mathrm{r}}\right), \tilde{B}_{\Omega_{\mathrm{s}}}$ from $L^{2}\left(\Omega_{\mathrm{s}}\right)$ to $V^{\infty}, \tilde{B}_{\mathrm{r}}$ from $L^{\infty}\left(\Gamma_{\mathrm{r}}\right)$ to $V^{\infty}$, and $\tilde{B}_{0}$ from $L^{\infty}\left(\Gamma_{\mathrm{r}}\right)$ to $V^{\infty}$. By the chain rule, $T$ is continuously Fréchet differentiable from $L^{2}\left(\Omega_{s}\right) \times V^{\infty}$ to $V^{\infty}$, and, in particular, continuous.

It remains to show the invertibility of $\frac{\partial T}{\partial y}(\bar{u}, \bar{y})$. For a given $f \in V^{\infty}$, we have to prove the existence of a unique $y \in V^{\infty}$ satisfying $\frac{\partial T}{\partial y}(\bar{u}, \bar{y}) y=f$, i.e., in view of (7.4),

$$
y+4 \tilde{B}_{\mathrm{r}}\left(G\left(\sigma|\bar{y}|^{3} y\right)\right)=f+\tilde{B}_{0}\left(\lambda y-4 \varepsilon \sigma|\bar{y}|^{3} y\right) .
$$

With the substitution $y=f-w \in V^{\infty}$, this is equivalent to the following weak formulation

$$
\begin{aligned}
\int_{\Omega} \kappa \nabla w \cdot \nabla v d x & +\int_{\Gamma_{\mathrm{r}}} 4 G\left(\sigma|\bar{y}|^{3} w\right) v d s+\int_{\Gamma_{0}} 4 \varepsilon \sigma|\bar{y}|^{3} w v d s \\
& =\int_{\Gamma_{\mathrm{r}}} 4 G\left(\sigma|\bar{y}|^{3} f\right) v d s-\int_{\Gamma_{0}}\left(\lambda-4 \varepsilon \sigma|\bar{y}|^{3}\right) f v d s \quad \forall v \in H^{1}(\Omega),
\end{aligned}
$$

whose bilinear form coincides with the one of (6.2). Here, the inhomogeneity is given by $f_{\Omega}=0, f_{0}:=\left(\varepsilon \sigma|\bar{y}|^{3}-\lambda\right) f \in L^{\infty}\left(\Gamma_{0}\right)$, and $f_{\mathrm{r}}:=4 G\left(\sigma|\bar{y}|^{3} f\right) \in L^{\infty}\left(\Gamma_{\mathrm{r}}\right)$. Thus, it fulfills the hypotheses of Theorems 6.1 and 6.2. We therefore have $w \in V^{\infty}$, and, hence, to every $f \in V^{\infty}$, there exists a unique solution $y \in V^{\infty}$, that yields the desired bijectivity of $\frac{\partial T}{\partial y}(\bar{u}, \bar{y})$.

Now, since all hypotheses are satisfied, we can apply the implicit function theorem to (7.4) and obtain, as derivative of $S$,

$$
y:=S^{\prime}(\bar{u})(u-\bar{u})=-\left(\frac{\partial T}{\partial y}(\bar{u}, \bar{y})\right)^{-1} \frac{\partial T}{\partial u}(\bar{u}, \bar{y})(u-\bar{u}) .
$$

Finally, a straightforward computation shows that $y$ satisfies (7.5) if, and only if, $y$ is the solution of the linearized equation (7.2).

7.2. The adjoint equation. A standard technique formally gives the following adjoint equation associated with $(\mathrm{P})$

$$
\begin{array}{rlrl}
\operatorname{div}\left(\kappa_{\mathrm{g}} \nabla p\right) & =\Delta \bar{y}-\operatorname{div} z & & \text { in } \Omega_{\mathrm{g}} \\
\operatorname{div}\left(\kappa_{\mathrm{s}} \nabla p\right) & =0 & & \text { in } \Omega_{\mathrm{s}} \\
\kappa_{\mathrm{S}}\left(\frac{\partial p}{\partial n_{\mathrm{r}}}\right)_{\mathrm{s}}-\kappa_{\mathrm{g}}\left(\frac{\partial p}{\partial n_{\mathrm{r}}}\right)_{\mathrm{g}}+4 \sigma|\bar{y}|^{3} G^{*} p & =\frac{\partial y}{\partial n_{\mathrm{r}}}-z \cdot n_{\mathrm{r}} & & \text { on } \Gamma_{\mathrm{r}} \\
\kappa_{s} \frac{\partial p}{\partial n_{0}}+4 \varepsilon \sigma|\bar{y}|^{3} p & =0 & & \\
& & \text { on } \Gamma_{0} .
\end{array}
$$


Formal integration by parts, also on the right-hand side, yields the corresponding weak formulation of (7.6):

$$
\begin{aligned}
\int_{\Omega} \kappa \nabla p \cdot \nabla v d x+4 & \int_{\Gamma_{\mathrm{r}}} \sigma|\bar{y}|^{3} G^{*}(p) v d s+4 \int_{\Gamma_{0}} \varepsilon \sigma|\bar{y}|^{3} p v d s \\
& =\int_{\Omega_{\mathrm{g}}}(\nabla \bar{y}-z) \cdot \nabla v d x=:\langle w, v\rangle \quad \forall v \in H^{1}(\Omega)
\end{aligned}
$$

with $w \in H^{1}(\Omega)^{*}$, since $\bar{y} \in V^{\infty}$ and $z \in L^{2}\left(\Omega_{\mathrm{g}}\right)$ by assumption (A3).

To show the existence of a unique solution to (7.7), we use a similar technique as for the linearized equation (6.1) based on the Fredholm alternative (see Section 6).

To that end, we transform (7.7) into

$$
\int_{\Omega} \kappa \nabla p \cdot \nabla v d x+4 \int_{\Gamma_{0}} \varepsilon \sigma|\bar{y}|^{3} p v d s=\langle w, v\rangle-4 \int_{\Gamma_{\mathrm{r}}} \sigma|\bar{y}|^{3} G^{*}(p) v d s .
$$

Due to the positivity of $\bar{y}$, the bilinear form defined by the left-hand side is bounded and coercive in $H^{1}(\Omega)$. Thus, we again obtain linear continuous operators $B_{\Omega}$ : $H^{1}(\Omega)^{*} \rightarrow H^{1}(\Omega)$ and $B_{\mathrm{r}}: L^{2}\left(\Gamma_{r}\right) \rightarrow H^{1}(\Omega)$ such that

$$
p=B_{\Omega} w+B_{\mathrm{r}}\left(-4 \sigma|\bar{y}|^{3} G^{*}\left(\tau_{\mathrm{r}} p\right)\right)
$$

(similar to (6.3), p. 13). Now we can argue as in the proof of Theorem 6.1 and, thus, obtain the following result:

ThEOREM 7.2. Assume $\bar{y} \in V^{\infty}, \bar{y} \geq \vartheta>0$, and that $\lambda=1$ is not an eigenvalue of $B(\bar{y})(\cdot):=-\tau_{\mathrm{r}} B_{\mathrm{r}}\left(4 \sigma|\bar{y}|^{3} G^{*}(\cdot)\right)$ with $B(\bar{y}): L^{2}\left(\Gamma_{\mathrm{r}}\right) \rightarrow L^{2}\left(\Gamma_{\mathrm{r}}\right)$. Then, to every $w \in H^{1}(\Omega)^{*}$, there exists a unique solution of $(7.7)$ in $H^{1}(\Omega)$.

Now, if we choose $v=p$ as test function in the weak formulation of the linearized equation (7.2), we obtain

$$
\int_{\Omega} \kappa \nabla y \cdot \nabla p d x+4 \int_{\Gamma_{\mathrm{r}}} G\left(\sigma|\bar{y}|^{3} y\right) p d s+4 \int_{\Gamma_{0}} \varepsilon \sigma|\bar{y}|^{3} y p d s=\int_{\Omega_{\mathrm{s}}}(u-\bar{u}) p d x .
$$

On the other hand, we insert $v=y$ in the weak formulation of the adjoint equation:

$$
\int_{\Omega} \kappa \nabla p \cdot \nabla y d x+4 \int_{\Gamma_{\mathrm{r}}} \sigma|\bar{y}|^{3} y G^{*}(p) d s+4 \int_{\Gamma_{0}} \varepsilon \sigma|\bar{y}|^{3} p y d s=\int_{\Omega_{\mathrm{g}}}(\nabla \bar{y}-z) \cdot \nabla y d x .
$$

Substracting one equation from the other yields $(\nabla \bar{y}-z, \nabla y)_{L^{2}\left(\Omega_{\mathrm{g}}\right)}=(u-\bar{u}, p)_{L^{2}\left(\Omega_{\mathrm{s}}\right)}$ for the first expression in the variational inequality (7.1). Thus, (7.1) can be transformed into

$$
j^{\prime}(\bar{u})(u-\bar{u})=\int_{\Omega_{\mathrm{s}}}(u-\bar{u})(p+\nu \bar{u}) d x \geq 0 \quad \forall u \in U_{\mathrm{ad}},
$$

and, finally, a standard pointwise discussion leads to the projection formula

$$
\bar{u}(x)=\mathcal{P}_{\left[u_{a}, u_{b}\right]}\left\{-\frac{1}{\nu} p(x)\right\},
$$

where $\mathcal{P}_{\left[u_{a}, u_{b}\right]}$ denotes the pointwise projection operator on $\left[u_{a}, u_{b}\right]$. 
8. Numerical tests. In this section, we report on some two-dimensional numerical tests. For the computational domain, we chose the square presented in Figure 1.1, p. 1 , which is naturally academic. In contrast to this, the material parameters were chosen to approximate the realistic distributions given in [11]. Two different temperature levels were investigated in our calculations. At the lower level, the temperature in the gas phase amounts to about $450 \mathrm{~K}$, whereas, at the higher level, it constitutes approximately $2000 \mathrm{~K}$ and, thus, is sufficiently high for the PVT method mentioned in Section 1. In the low-temperature example, the thermal conductivity ${ }^{2}$ in the gas phase is fixed at $\kappa_{\mathrm{g}} \equiv 0.03$ and, having in mind a graphite crucible, at $\kappa_{\mathrm{s}} \equiv 35.0$ in the solid. At the higher temperatures, we obtain $\kappa_{\mathrm{g}} \equiv 0.08$ and $\kappa_{\mathrm{S}} \equiv 24.0$. The emissivity is set to $\varepsilon \equiv 0.65$ at the lower temperature level and $\varepsilon \equiv 0.8$ in the high temperature case. In all cases, the Boltzmann radiation constant ${ }^{3}$ is given by $\sigma=5.6696 \cdot 10^{-8}$ and the external temperature by $y_{0}=293.0 \mathrm{~K}$.

As this paper is concerned with first order sensitivity analysis, we implemented a gradient type method to be consistent with the theory. More precisely, we used a projected gradient method with a line search according to the Armijo rule. Although the projected gradient method needs a quite large number of iterations, each step is comparatively cheap and fast. The only time-consuming part of each iteration is to solve the semilinear equation which was done by the Newton method. The linearized PDE and the adjoint equation were approximated by linear finite elements. The integral operators $G$ and $K$ arising from the nonlocal radiation condition on $\Gamma_{\mathrm{r}}$ were discretized by a summarized midpoint rule.

We present three numerical examples. In the first example, at the lower temperature level, the optimal temperature profile nearly reaches the desired temperature gradient. The other two examples refer to the higher temperature level. In these cases, the desired temperature gradient is hardly been achieved. This applies especially to the last example, where the optimal control is almost bang-bang, since the desired temperature gradient is quite large compared to the bounds $u_{a}$ and $u_{b}$.

In the first example, the desired temperature gradient ${ }^{4}$ was given by $z=(0,20)^{T}$, and we took $u_{a}=2000$, and $u_{b}=8000$ for the control constraints ${ }^{5}$. To compensate for the comparatively large values of the control, we choose a small Tikhonov regularization parameter $\nu=5 \cdot 10^{-7}$. Because of the bounds for the heat sources, the average temperature in the gas phase is significantly lower than in the other examples. The pictures 8.1-8.4 show the optimal control, state, and adjoint state, calculated by the projected gradient method. The optimal control shown in Fig. 8.1 is only defined in the solid parts $\Omega_{\mathrm{s}}$, and, therefore, its graph shows a hole in the inner square, where the gas phase is located.

\footnotetext{
${ }^{2}$ in $\mathrm{W} /(\mathrm{m} \mathrm{K})$

${ }^{3}$ in $\mathrm{W} /\left(\mathrm{m}^{2} \mathrm{~K}^{4}\right)$

${ }^{4}$ in $\mathrm{K} / \mathrm{m}$

${ }^{5}$ in W/m ${ }^{3}$
} 


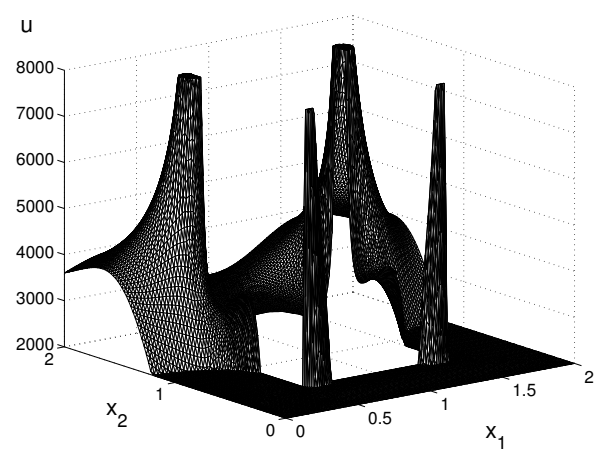

FIG. 8.1. Control $u$ in the first example.

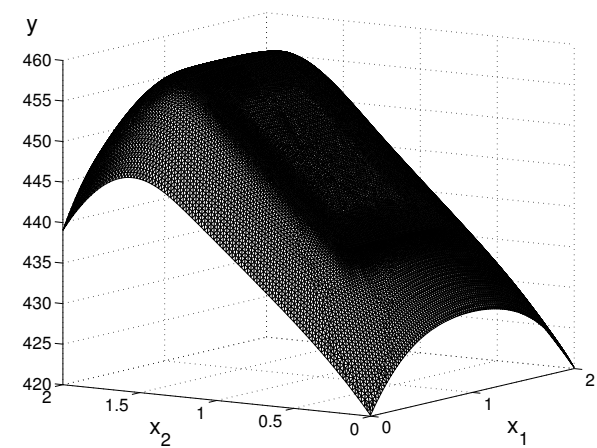

FIG. 8.3. State $y$ in the first example.

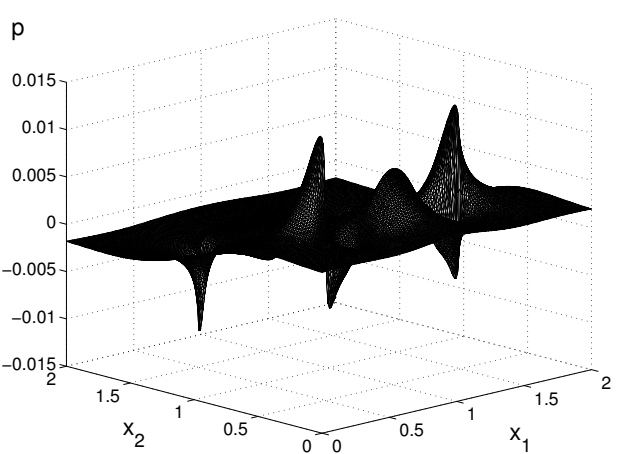

FIG. 8.2. Adjoint state $p$ in the first example.

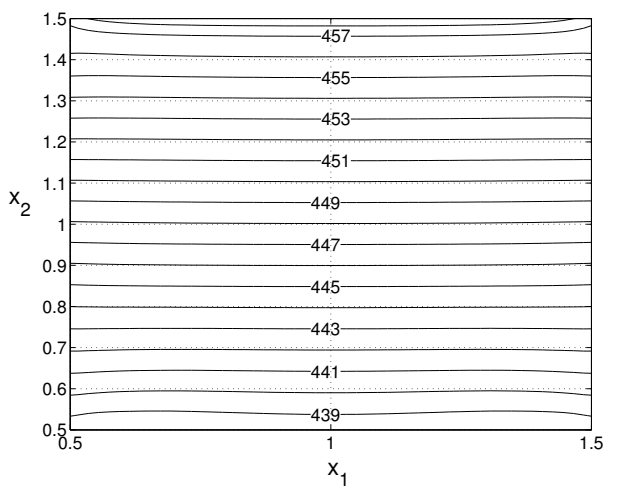

FIG. 8.4. Isotherms in the gas phase $\Omega_{\mathrm{g}}$.

As one can see in Fig. 8.4, the desired temperature gradient is nearly reached. Furthermore, the control $u$ possesses peaks in the corners of the inner boundary $\Gamma_{\mathrm{r}}$. As we observed the same behavior in calculations on two different meshes with 2705 and 16474 grid points, respectively, this does not seem to show a numerical effect. A possible explanation is that the temperature tends to decrease in the corners, as one can see in the other two examples, where the optimal temperature gradient differs significantly from the desired one. Since a constant temperature distribution in the $x_{1}$-direction is required, the optimal control must increase in the corners to compensate for the decrease of the temperature.

To deal with higher temperatures in the gas phase, we now set $u_{a}=125000$ and $u_{b}=$ 725000. Accordingly, the Tikhonov regularization parameter is reduced to $\nu=3 \cdot 10^{-9}$. As in the first example, the desired temperature gradient is given by $z=(0,20)^{T}$. The following pictures show the optimal control and state in the gas phase for this setting. 


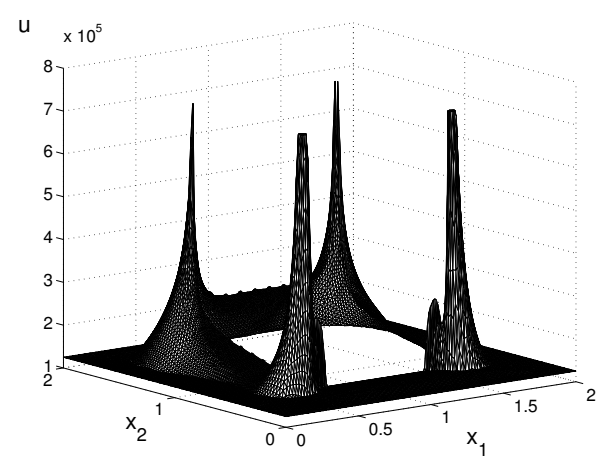

FIG. 8.5. Control $u$ in the second example.

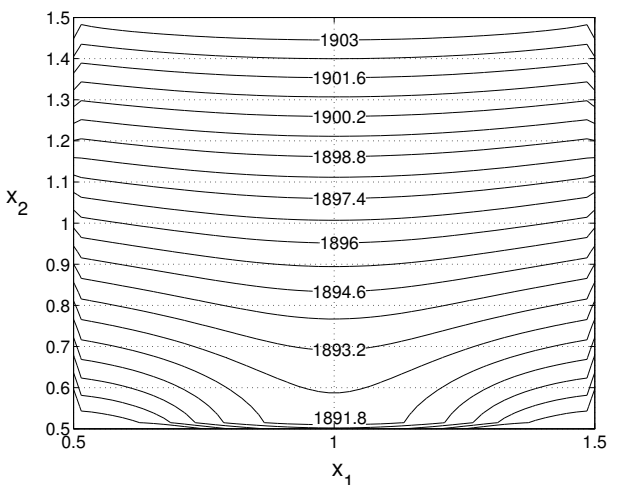

FIG. 8.6. Isotherms in the gas phase $\Omega_{\mathrm{g}}$.

In this example, the optimal temperature distribution in the gas phase differs considerably from the desired temperature gradient. The difference between the temperature at $x_{2}=0.5$ and $x_{2}=1.5$ amounts to about $14 \mathrm{~K}$ and, thus, is significantly smaller than the desired value of $20 \mathrm{~K}$. Furthermore, the temperature is no longer constant in the $x_{1}$-direction.

This behavior especially occurs in the corners, although we again obtain the peaks of the control $u$ in these corners, as one can see in Fig. 8.5. A possible explanation for this result is the strong cooling effect due to the relatively low external temperature. Because of the comparatively large difference of about $1700 \mathrm{~K}$ between the temperature in the gas phase and the external temperature outside the crucible, one obtains quite steep gradients in the solid part $\Omega_{\mathrm{s}}$, as one can see in Fig. 8.7. Therefore, it is no longer possible to generate a temperature distribution

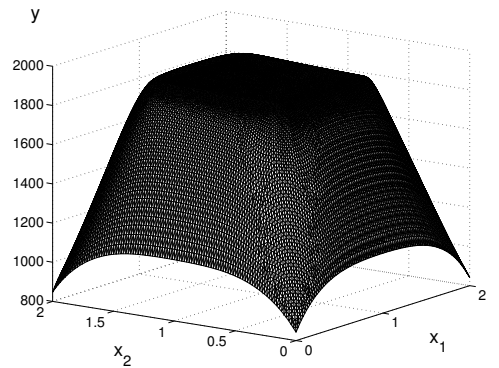

FIG. 8.7. State $y$ in the second example. that is constant in the $x_{1}$-direction.

This behavior is even more pronounced in the third example, as one can see in Fig. 8.9. In this example, we set $u_{a}=200000, u_{b}=300000$, and $z=(0,100)^{T}$. As indicated at the beginning of this section, the desired $x_{2}$-derivative $z_{2}$ is comparatively steep and, therefore, cannot be achieved with these bounds on the control. Thus, the optimal control is almost bang-bang, as Fig. 8.8 shows. 


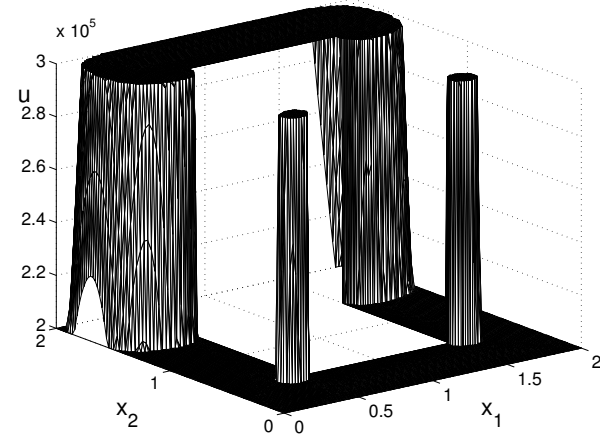

FIG. 8.8. Control $u$ in the third example.

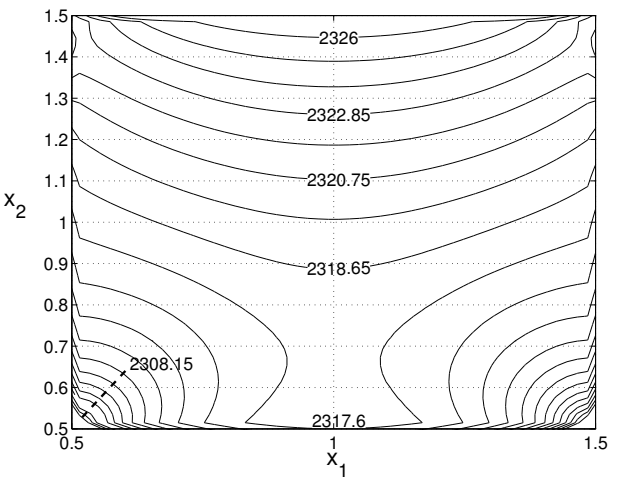

FIG. 8.9. Isotherms in the gas phase $\Omega_{\mathrm{g}}$.

\section{REFERENCES}

[1] J. Bonnans, Second order analysis for control constrained optimal control problems of semilinear elliptic systems, Appl. Math. Optimization, 38 (1998), pp. 303-325.

[2] J. Bonnans, E. CASAS, Une principe de Pontryagine pour le contrôle des systèmes semilinéaires elliptiques, J. Diff. Equations, 90 (1991), pp. 288-303.

[3] E. CAsas, Pontryagin's principle for optimal control problems governed by semilinear elliptic equations, International Series of Numerical Mathematics, 118 (1994), pp. 97-114.

[4] E. Casas And M. Mateos, Second Order sufficient optimality conditions for semilinear elliptic control problems with finitely many state constraints, SIAM J. Control and Optimization, 40 (2002), pp. 1431-1454.

[5] E. CAsAs, F. Tröltzsch, AND A. Unger, Second order sufficient optimality conditions for a nonlinear elliptic control problem J. for Analysis and its Applications 15 (1996), pp. 687707.

[6] D. Kinderlehrer and G. Stampacchia, An Introduction to Variational Inequalities and their Applications, Vol. 88 of Pure and Applied Mathematics, Academic Press, New York, 1980.

[7] O. Klein, P. Philip, And J. Sprekels, Modeling and simulation of sublimation growth of SiC bulk single crystals, Interfaces and Free Boundaries, 6 (2004), pp. 295-314.

[8] A. Konstantinov, Sublimation growth of SiC, Ch. 8.2, pp. 170-203 in G. Harris, ed., Properties of Silicon Carbide, No. 13 in EMIS Datareview Series, Institution of Electrical Engineers, INSPEC, London, UK, 1995.

[9] M. A. Krasnoselskit, P. P. Zabreiko, E. N. Pustylnik and P. E. Sobolevskil, Integral Operators in Spaces of Summable Functions, Leyden, Noordho, 1976.

[10] M. Laitinen and T. Tilhonen, Conductive-radiative heat transfer in grey materials, Quart. Appl. Math., 59 (2001), pp. 737-768.

[11] P. PHILIP, Transient Numerical Simulation of Sublimation Growth of SiC Bulk Single Crystals. Modeling, Finite Volume Method, Results, PhD thesis, Department of Mathematics, Humboldt University of Berlin, Germany, 2003. Report No. 22, Weierstrass Institute for Applied Analysis and Stochastics, Berlin.

[12] H.-J. Rost, D. Siche, J. Dolle, W. Eiserbeck, T. Müller, D. Schulz, G. Wagner, and $\mathrm{J}$. WOLLWEBER, Influence of different growth parameters and related conditions on $6 \mathrm{H}$-SiC crystals grown by the modified Lely method, Mater. Sci. Eng. B, 61-62 (1999), pp. 68-72.

[13] G. Stampacchia, Le problme de dirichlet pour les quations elliptiques du second ordre coeffcients discontinus, Ann. Inst. Fourier, Grenoble, 15 (1965), pp. 189-258.

[14] T. Tinhonen, A nonlocal problem arising from heat radiation on non-convex surfaces, Eur. J. App. Math., 8 (1997), pp. 403-416.

[15] _ Stefan-Boltzmann radiation on non-convex surfaces, Math. Meth. in Appl. Sci., 20 (1997), pp. 47-57.

[16] E. ZeIDLER, Nonlinear Functional Analysis and its Applications, Vol. II/B, Springer-Verlag, New York, 1990. 